Monika Thol, Muhammad Ali Javed, Elmar Baumhögger, Roland Span, Jadran Vrabec

\title{
Thermodynamic Properties of Dodecamethylpentasiloxane, Tetradecamethylhexasiloxane, and Decamethylcyclopentasiloxane
}

Journal article | Accepted manuscript (Postprint)

This version is available at https://doi.org/10.14279/depositonce-9122

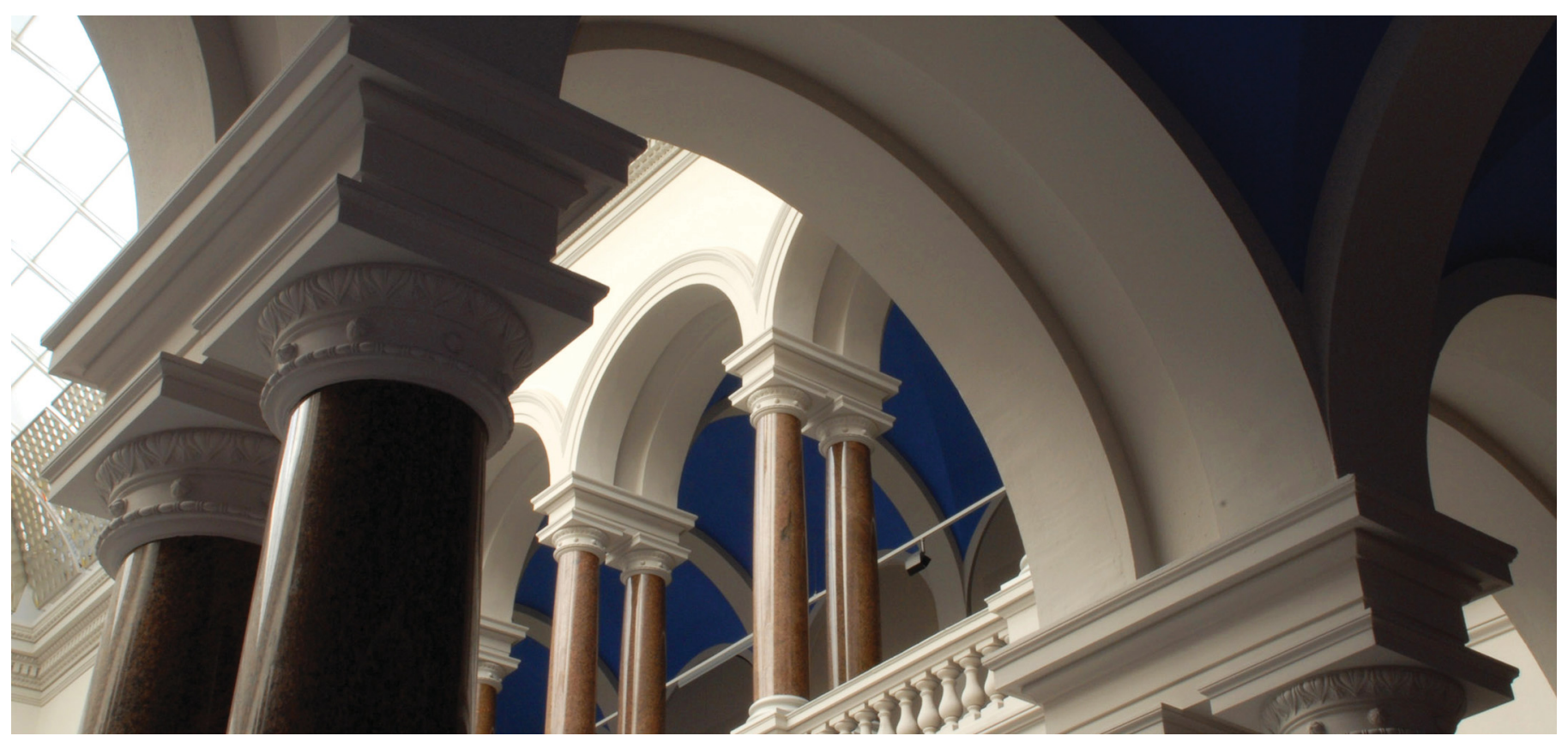

Thol, M., Javed, M. A., Baumhögger, E., Span, R., \& Vrabec, J. (2019). Thermodynamic Properties of Dodecamethylpentasiloxane, Tetradecamethylhexasiloxane, and Decamethylcyclopentasiloxane. Industrial \& Engineering Chemistry Research, 58(22), 9617-9635. https://doi.org/10.1021/acs.iecr.9b00608 


\title{
Thermodynamic properties of Dodecamethylpentasiloxane, Tetradecame- thylhexasiloxane, and Decamethylcyclopentasiloxane
}

\author{
Monika Thol $^{1 *}$, Muhammad Ali Javed ${ }^{2}$, Elmar Baumhögger ${ }^{3}$, Roland Span ${ }^{1}$, Jadran Vrabec ${ }^{2}$ \\ ${ }^{1}$ Lehrstuhl für Thermodynamik, Ruhr-Universität Bochum, Universitätsstraße 150, \\ 44801 Bochum, Germany \\ ${ }^{2}$ Thermodynamik und Thermische Verfahrenstechnik, Technische Universität Berlin, \\ Ernst-Reuter-Platz 1, 10587 Berlin, Germany \\ ${ }^{3}$ Lehrstuhl für Thermodynamik und Energietechnik, Universität Paderborn, Warburger \\ Straße 100, 33098 Paderborn, Germany
}

\begin{abstract}
Siloxanes are widely used in the chemical industry and in process and power engineering. For example, since 20 years, they are used as working fluids of organic Rankine cycle power plants. For the process design and optimization, thermodynamic properties, such as enthalpy, entropy, speed of sound, density, and vapor-liquid equilibrium, are required. While the properties of short-chained siloxanes, such as hexamethyldisiloxane (MM) or octamethylcyclotetrasiloxane $\left(\mathrm{D}_{4}\right)$, have already been investigated comprehensively, information on thermophysical properties of higher order siloxanes is limited. Therefore, measurements on density and speed of sound in the liquid state of dodecamethylpentasiloxane $\left(\mathrm{MD}_{3} \mathrm{M}\right)$, tetradecamethylhexasiloxane $\left(\mathrm{MD}_{4} \mathrm{M}\right)$, and decamethylcyclopentasiloxane $\left(\mathrm{D}_{5}\right)$ are presented here. Based on these measurements and other experimental data from the literature, new fundamental equations of state were developed for these three fluids. The equations are based on the Helmholtz energy and, thus, allow for the calculation of any thermodynamic state property by means of derivatives with respect to the natural variables, namely temperature and density. The obtained models also feature a correct extrapolation behavior in regions where no data are available in order to ensure the applicability of the equations to mixture models in the future.
\end{abstract}

Based on the present equations of state and recently published equations for other siloxanes, the possible belonging of the siloxanes to the group of Bethe-Zel'dovich-Thompson fluids is also investigated.

*E-mail: M.Thol@thermo.ruhr-uni-bochum.de 


\section{INTRODUCTION}

The investigation documented in this paper is part of a comprehensive study on the thermodynamic properties of linear and cyclic siloxanes. Siloxanes are used extensively in chemistry and process engineering, e.g., for hygiene articles, cleaning agents, cosmetics, polishing agents, solvents, and as a petrol additive. ${ }^{1-4}$

Apart from commercial use, there is much scientific interest in accurate properties of siloxanes with regard to the study of non-classical phenomena of gas dynamics. These phenomena are unusual flow features that are predicted to occur in compressible flows of rather complex organic molecules close to saturation and to the vapor-liquid critical point. Such fluids are called Bethe-Zel'dovich-Thompson (BZT) fluids. ${ }^{5-7}$ In the last decades, the interest in this group of substances grew because they are expected to be suitable working fluids of high-temperature organic Rankine cycles (ORC). Since they possibly belong to the class of BZT fluids, they might allow a particularly efficient operation of such processes. ${ }^{8}$ While short-chained molecules of the siloxane family are used in mainstream ORC power plants, the three siloxanes investigated in this work might be interesting for future applications, such as micro-power plans. ${ }^{9,10}$ Furthermore, siloxane mixtures are considered to be attractive for the mainstream high-temperature power plants. ${ }^{9}$ The main reason for selecting siloxanes as working fluid in ORC processes are high thermal stability, suitable thermodynamic and transport properties, low flammability, low toxicity, availability, and comparatively low costs. ${ }^{10,11}$ Furthermore, siloxanes are components of liquid mixtures that are used as heat transfer fluids in combination with industrial boilers or in solar thermal power plants. ${ }^{12,13}$

For the efficient design and optimization of processes, thermodynamic properties are required. Such properties are provided by fundamental equations of state, as were developed for these fluids by Colonna et al. ${ }^{14,15}$ in 2006 and 2008. In these publications, equations of state in terms of the Helmholtz energy were presented for several linear and cyclic siloxanes. A decade ago, however, experimental information on the thermodynamic properties of these fluids was rather limited so that Colonna et al. ${ }^{14,15}$ employed a generalized functional form for non-polar fluids as proposed by Span and Wagner. ${ }^{16}$ For the characterization of individual siloxanes, only the coefficients were adjusted to the available experimental data as opposed to optimizing also the functional form. This is a well-established procedure if only limited experimental information about the corresponding fluid is available, but such models provide thermodynamic properties with limited accuracy. In 2011, Abbas et al. ${ }^{17}$ carried out a comprehensive study on the liquid density of several siloxanes. Based on these results and supplemented by additional measurements on the speed of sound in the liquid state, the generalized equations for hexamethyldisiloxane (MM), octamethyltrisiloxane (MDM), decamethyltetrasiloxane $\left(\mathrm{MD}_{2} \mathrm{M}\right)$, and octamethylcyclotetrasiloxane $\left(\mathrm{D}_{4}\right)$ were superseded by more accurate fluid-specific equations 
of state. ${ }^{18-20}$ These models also allow for a much more reliable assessment to determine if these fluids belong to the BZT fluid class. BZT fluids are characterized by negative values of the socalled fundamental derivative of gas dynamics along the saturated vapor curve in the nearcritical region, which is a secondary thermodynamic property depending on the variation of the speed of sound with density at constant entropy. For the four siloxanes for which revised equations of state are already available, it was shown that the correlations of Colonna et al. ${ }^{14,15}$ deviate from the new experimental speed of sound data by more than $15 \%$. The revised equations of state reproduce these data within approximately $0.3 \%$ to $0.5 \%$ and, therefore, also provide more reliable information on the fundamental derivative of gas dynamics. Based on these equations, it was shown that $\mathrm{MM}, \mathrm{MDM}, \mathrm{MD}_{2} \mathrm{M}$, and $\mathrm{D}_{4}$ do not belong to the $\mathrm{BZT}$ fluid class because they exhibit positive values along the entire saturated vapor line up to the critical point.

Experimental data on the liquid density, speed of sound, and vapor pressure are important for the development of this type of equations of state. Furthermore, information on the isobaric heat capacity of ideal-gas states is required to set up the ideal part of the equation of state, which is needed for the calculation of caloric properties. For the siloxanes that are in the focus of this work, only vapor-pressure data and rather inaccurate information on the ideal-gas heat capacities are available in the literature. Therefore, speed of sound measurements presented in this work were measured with the same dual-path pulse-echo system as previously described. ${ }^{18-22}$ Since Abbas et al. ${ }^{17}$ did not measure the liquid density of these fluids, additional experimental work on this property was required. For this purpose, an Anton Paar vibrating tube densimeter (type DMA HPM) was applied in this work to measure the density of the liquid phase.

Based on these measurements and additional experimental information from the literature, new fluid-specific fundamental equations of state in terms of the Helmholtz energy were developed for dodecamethylpentasiloxane $\left(\mathrm{MD}_{3} \mathrm{M}\right.$, chemical formula: $\mathrm{C}_{12} \mathrm{H}_{36} \mathrm{O}_{4} \mathrm{Si}_{5}$ ), tetradecamethylhexasiloxane $\left(\mathrm{MD}_{4} \mathrm{M}\right.$, chemical formula: $\left.\mathrm{C}_{14} \mathrm{H}_{42} \mathrm{O}_{5} \mathrm{Si}_{6}\right)$, and decamethylcyclopentasiloxane (D , chemical formula: $\mathrm{C}_{10} \mathrm{H}_{30} \mathrm{O}_{5} \mathrm{Si}_{5}$ ). For the sake of completeness, the development of a new equation of state for dodecamethylcyclohexasiloxane $\left(D_{6}\right)$ was also planned. However, it was not possible to obtain a sample with adequate purity at an affordable cost so that measurements of sufficient quality could not be carried out. Hence, for this fluid no improvement regarding its thermodynamic property modeling could be realized in comparison to the model documented by Colonna et al. ${ }^{15}$ 


\section{DENSITY MEASUREMENTS IN THE LIQUID STATE}

An U-shaped vibrating tube densimeter (Anton Paar, DMA HPM) was used to measure the density of the fluids, see Figure 1. This device consists of two main components, a measurement cell with an oscillator and an interface module, which generates and measures the oscillation as well as the cell temperature. The working principle of the densimeter is based on the relation between the density of the fluid and the frequency of its oscillations once it is put into motion by an actuator. Therefore, the density

$$
\rho=\rho(T, p, s),
$$

is a function of temperature $T$, pressure $p$, and oscillation period $s$. To calculate the density from the measured oscillation period, a calibration method as suggested by Anton Paar $^{23}$ was followed. For this purpose, water and ethanol were selected as reference fluids because their density is similar to that of the siloxanes and high quality equations of state are available for these standard fluids. ${ }^{24,25}$

The oscillation period was fitted over the entire temperature and pressure range with a fourthorder Legendre polynomial with 20 coefficients by considering the individual parameters $(T, p$, and $s$ ) and their possible combinations. After excluding terms with weak contributions or high uncertainties, a third-order equation with 12 coefficients was found

$$
\begin{aligned}
\rho= & a+b_{1} \tilde{T}+c_{1} \tilde{p}+d_{1} \tilde{s}+b_{2} \frac{\left(3 \tilde{T}^{2}-1\right)}{2}+d_{2} \frac{\left(3 \tilde{s}^{2}-1\right)}{2}+b_{1} d_{1} \tilde{T} \tilde{s}+b_{1} d_{2} \tilde{T} \frac{\left(3 \tilde{s}^{2}-1\right)}{2} \\
& +c_{1} d_{2} \tilde{p} \frac{\left(3 \tilde{s}^{2}-1\right)}{2}+d_{1} b_{2} \tilde{s} \frac{\left(3 \tilde{T}^{2}-1\right)}{2}+d_{1} c_{2} \tilde{s} \frac{\left(3 \tilde{p}^{2}-1\right)}{2}+b_{1} c_{1} d_{1} \tilde{T} \tilde{p} \tilde{s} .
\end{aligned}
$$

Coefficients $a, b_{i}, c_{i}$, and $d_{i}$ (parameters are listed in Table 1 in the Supplementary Material) were adjusted to calibration data of the reference fluids, and $\tilde{T}, \tilde{p}$, and $\tilde{s}$ are the scaled temperature, pressure, and oscillation period in the sense

$$
\tilde{y}=\frac{y_{\text {mean }}}{\text { width }_{y}} .
$$

The siloxanes were purchased with the purities listed in Table 1. In the present work, the sample fluids were degassed by keeping the siloxanes at ambient temperature under vacuum for $24 \mathrm{~h}$. The vapor pressure of the siloxanes is very low so that possible water traces have mostly been mitigated by evacuation.

Table 1. Specification of the siloxanes.

\begin{tabular}{lllccc}
\hline chemical name & short name & CAS no. & chemical formula & purity / \% & source \\
\hline dodecamethylpentasiloxane & $\mathrm{MD}_{3} \mathrm{M}$ & $141-63-9$ & $\mathrm{C}_{12} \mathrm{H}_{36} \mathrm{O}_{4} \mathrm{Si}_{5}$ & 99.7 & Sigma-Aldrich \\
tetradecamethylhexasiloxane & $\mathrm{MD}_{4} \mathrm{M}$ & $107-52-8$ & $\mathrm{C}_{14} \mathrm{H}_{42} \mathrm{O}_{5} \mathrm{Si}_{6}$ & $>95$ & CHEMOS \\
decamethylcyclopentasiloxane & $\mathrm{D}_{5}$ & $541-02-6$ & $\mathrm{C}_{10} \mathrm{H}_{30} \mathrm{O}_{5} \mathrm{Si}_{5}$ & $\geq 96.5$ & Sigma-Aldrich \\
\hline
\end{tabular}


To carry out measurements, the evacuated cell was filled with sample fluid. The densimeter was surrounded by a liquid jacket in combination with an external thermostat to ensure a stable measuring temperature within $0.01 \mathrm{~K}$, cf. Figure 1 . Water was used for thermostating in the temperature range between $274 \mathrm{~K}$ and $364 \mathrm{~K}$. This range is limited to $10 \mathrm{~K}$ below the normal boiling point of water in order to avoid damage of the circulating pump or electrical circuits due to excessive evaporation. The cell temperature $T$ and oscillation period $s$ were directly gathered from the DMA HPM built-in interface module with uncertainties of $0.2 \mathrm{~K}$ and $0.06 \mu \mathrm{s}$, respectively. A hand pump (HIP 50-6-15) was used to specify a pressure of up to $100 \mathrm{MPa}$. To measure this property, a pressure transducer (Keller PAA-33X) with an uncertainty of $0.04 \mathrm{MPa}$ was used.

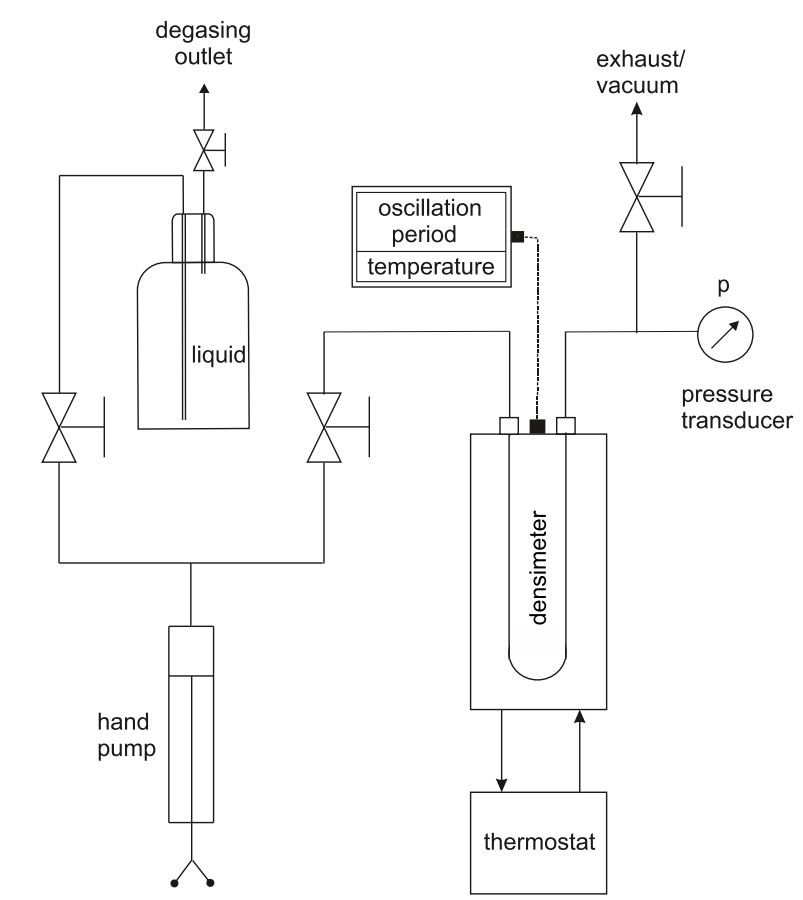

Figure 1. Experimental setup for measuring the specific density of the siloxanes.

The overall expanded uncertainty of the density $U_{\rho}$ was calculated at a confidence level of $95 \%$ (coverage factor $k=2$ ) from the individual standard uncertainties due to temperature $u_{T}$, pressure $u_{p}$, oscillation period $u_{s}$, and calibration of the measurement cell $u_{\text {cal }}$ as

$$
U_{\rho}=2 \sqrt{\left(\left(\frac{\partial \rho}{\partial T}\right)_{p, s} u_{T}\right)^{2}+\left(\left(\frac{\partial \rho}{\partial p}\right)_{T, s} u_{p}\right)^{2}+\left(\left(\frac{\partial \rho}{\partial s}\right)_{p, T} u_{s}\right)^{2}+u_{\mathrm{cal}}^{2}}
$$

The partial derivatives of density with respect to temperature and pressure were calculated from the present equations of state, whereas the derivative with respect to the oscillation period was determined from experimental data. The density of the siloxanes was measured along seven isotherms with an overall expanded uncertainty $(k=2)$ below $0.11 \%$ for the entire temperature and pressure range. Experimental density data with uncertainties are provided in numerical 
form in Tables 2 to 4 in the Supplementary Material. Since the molecular species of the impurities of the samples could not be identified, it was not possible to include their contribution into the uncertainty analysis. However, due to the production process, the main impurities are adjacent species of the actual siloxane. ${ }^{26}$ Since the thermodynamic properties of these siloxanes are similar (cf. Tables 2 to 4 and 6 to 8 in the Supplementary Material), the actual uncertainty of the present measurements is expected to be only slightly higher than the numbers given in the tables. 


\section{SPEED OF SOUND MEASUREMENTS}

Speed of sound measurements for the siloxanes were performed with a double path-length pulse-echo technique, combined with burst design and signal processing. ${ }^{21,22} \mathrm{~A} X$-cut piezoelectric quartz crystal with a resonance frequency of $8 \mathrm{MHz}$ is positioned asymmetrically between two metallic reflectors located at distances of $l_{1} \approx 20 \mathrm{~mm}$ and $l_{2} \approx 30 \mathrm{~mm}$. The quartz crystal is excited electrically with a sinusoidal burst signal of eight periods. Consequently, two sound waves are emitted and propagated in opposite directions through the sample fluid. After reflection, they interact with the quartz crystal, which also operates as a receiver of the acoustic signals. These echoes are analyzed with a high-resolution oscilloscope and the propagation time of the two echoes is identified. The speed of sound is calculated as the ratio between the pathlength difference and the time of flight, neglecting dispersion and diffraction effects, as

$$
w=\frac{2\left(l_{2}-l_{1}\right)}{\Delta t} .
$$

To determine the path-length difference $\Delta l=2\left(l_{2}-l_{1}\right)$, the acoustic cell was calibrated by means of measurements of the speed of sound in water, for which highly accurate measurements are available. ${ }^{27-29}$ For the calculation of the speed of sound according to Eq. (5), path-length corrections to consider thermal expansion and pressure compression of the acoustic cell were applied. $^{5}$

The acoustic cell was mounted in a pressure vessel, which was surrounded by three copper jackets equipped with electrical heaters for thermostating. The entire setup was placed into a vacuum chamber to suppress convective heat losses. A detailed layout of the experimental rig was already provided by Thol et al. ${ }^{30}$ However, in the present work, more accurate temperature and pressure transducers were employed in the experimental campaign.

Prior to the measurements, the pressure vessel was evacuated for almost two hours. The sample was kept under vacuum at ambient temperature for almost 24 hours, while the vacuum was renewed several times. Subsequently, the degassed sample fluid was filled into the cell. The speed of sound was measured along seven isotherms between $218 \mathrm{~K}$ and $500 \mathrm{~K}$. For each isotherm, a hand pump (HIP 50-5.75-30) was used to compress the sample fluid stepwise up to a pressure of $125 \mathrm{MPa}$ and after an equilibration time of one hour a constant pressure level was attained.

The fluid temperature was measured with a PT-25 thermometer (type 162CE Rosemount) located in the wall of the pressure vessel with an estimated uncertainty of $0.04 \mathrm{~K}$. For pressure measurement, three pressure transducers were used (measurement range and uncertainty): Keller PAA-33X ( $\left.<10 \mathrm{MPa}, u_{p}=0.004 \mathrm{MPa}\right)$; Keller PAA-33X $\left(<100 \mathrm{MPa}, u_{p}=0.04 \mathrm{MPa}\right)$ and Honeywell TJE $\left(<200 \mathrm{MPa}, u_{p}=0.12 \mathrm{MPa}\right)$. The acoustic signals were analyzed with a USB oscilloscope (handyscope HS5) featuring an uncertainty of $u_{\Delta t}=0.004 \mu$ s for time measurement. The path-length difference of the acoustic cell was calibrated with an uncertainty of 
$u_{\Delta l}=14 \mu \mathrm{m}$. The overall expanded uncertainty of the speed of sound $U_{w}$ with a confidence level of $95 \%$ (coverage factor, $k=2$ ) was calculated from the contributions of individual standard uncertainties due to temperature $u_{T}$, pressure $u_{p}$, time of flight $u_{\Delta t}$, and path-length difference $u_{\Delta l}$ as

$$
U_{w}=2 \sqrt{\left(\left(\frac{\partial w}{\partial T}\right)_{p, \Delta t, \Delta l} u_{T}\right)^{2}+\left(\left(\frac{\partial w}{\partial p}\right)_{T, \Delta t, \Delta l} u_{p}\right)^{2}+\left(\left(\frac{\partial w}{\partial \Delta t}\right)_{T, p, \Delta l} u_{\Delta t}\right)^{2}+\left(\left(\frac{\partial w}{\partial \Delta l}\right)_{T, p, \Delta t} u_{\Delta l}\right)^{2}} \cdot(6)
$$

The partial derivatives of speed of sound with respect to temperature and pressure were calculated from the present equations of state, whereas the derivatives with respect to time and length were calculated from Eq. (5). Speed of sound data for the siloxanes were measured with an overall expanded uncertainty below $0.08 \%$ and are provided in numerical form in Tables 6 to 8 in the Supplementary Material.

Since the molecular species of the impurities of the samples could not be identified, it was not possible to include their contribution into the uncertainty analysis. Thus, the actual uncertainty is expected to be higher than the numbers given in the tables, see section 2. 


\section{EQUATIONS OF STATE}

Modern equations of state are expressed in terms of the Helmholtz energy with temperature $T$ and density $\rho$ being the independent variables. It is common practice to apply a dimensionless form of the Helmholtz energy $\alpha$ reduced by the temperature and the universal gas constant $R=8.3144598 \mathrm{~J} \cdot \mathrm{mol}^{-1} \cdot \mathrm{K}^{-1}$ as recommended by Mohr et al.$^{31}$

$$
\alpha(\tau, \delta)=\frac{a(T, \rho)}{R T}
$$

Subsequently, it is separated into an ideal part $\alpha^{\mathrm{o}}$ and a residual contribution $\alpha^{\mathrm{r}}$

$$
\alpha(\tau, \delta)=\alpha^{\mathrm{o}}(\tau, \delta)+\alpha^{\mathrm{r}}(\tau, \delta)
$$

with $\tau=T_{\mathrm{c}} / T$ and $\tau=\rho / \rho_{\mathrm{c}}$.

The ideal part expresses the contribution of the hypothetical ideal gas, whereas the residual part accounts for the deviation of the real fluid from this idealized concept. The approach of a static rotator and a harmonic oscillator as explained by $\mathrm{Span}^{32}$ considers energetic contributions of translation, rotation, and internal molecular vibrations for the ideal part of Eq. (8). For simplicity, translation and rotation are assumed to be always excited, except at very low temperatures $(T \rightarrow 0 \mathrm{~K})$. The temperature dependent vibration modes are represented by the PlanckEinstein terms for the isobaric heat capacity of the ideal gas

$$
\frac{c_{p}^{\mathrm{o}}}{R}=n_{0}+\sum_{i=1}^{3} m_{i}\left(\frac{\theta_{i}}{T}\right)^{2} \frac{\exp \left(\theta_{i} / T\right)}{\left(\exp \left(\theta_{i} / T\right)-1\right)^{2}} .
$$

For complex molecules like siloxanes, translation and rotation contribute to the isochoric heat capacity with three degrees of freedom each. With $c_{p}^{0}=c_{v}^{0}+R$, the temperature independent parameter is $n_{0}=4$. A two-fold integration of Eq. (9) with respect to the temperature yields the ideal-gas Helmholtz energy

$$
\alpha^{\mathrm{o}}(\tau, \delta)=c^{\mathrm{II}}+c^{\mathrm{I}} \tau+3 \ln (\tau)+\ln (\delta)+\sum_{i=1}^{3} m_{i} \ln \left(1-\exp \left(-\theta_{i} / T_{\mathrm{c}} \tau\right)\right)
$$

The corresponding parameters are listed in Table 2 .

Table 2. Parameters of the ideal part of the present equations of state for $\mathrm{MD}_{3} \mathrm{M}, \mathrm{MD}_{4} \mathrm{M}$, and $\mathrm{D}_{5}$ (cf. Eq. (10)).

\begin{tabular}{lccccc}
\hline$i$ & 1 & 2 & 3 & $c^{\mathrm{I}}$ & $c^{\mathrm{II}}$ \\
\hline$m_{i}$ & 81.2386 & 61.191 & 51.1798 & & \\
$\theta_{i} / \mathrm{K}$ & 610 & 2500 & 7500 & -29.8091965426 & 68.1167204166 \\
\hline \multicolumn{7}{c}{$\mathrm{MD}_{3} \mathrm{M}$} & & & \\
$m_{i}$ & 97.16 & 69.73 & 38.43 & & \\
$\theta_{i} / \mathrm{K}$ & 610 & 2480 & 6400 & & \\
\hline
\end{tabular}




\begin{tabular}{|c|c|c|c|c|c|}
\hline$i$ & 1 & 2 & 3 & $c^{\mathrm{I}}$ & $c^{\mathrm{II}}$ \\
\hline \multicolumn{6}{|c|}{$\mathrm{D}_{5}$} \\
\hline$m_{i}$ & 51.0 & 57.9 & 35.0 & \multirow{2}{*}{-31.1102222402} & \multirow{2}{*}{94.3892428631} \\
\hline$\theta_{i} / \mathrm{K}$ & 221 & 1733 & 4544 & & \\
\hline
\end{tabular}

The integration constants $c^{\mathrm{I}}$ and $c^{\mathrm{II}}$ are specified such that enthalpy and entropy are zero at the normal boiling point.

The parameters of the ideal-gas contribution $\left(m_{i}\right.$ and $\left.\theta_{i}\right)$ are usually adjusted to either measured or theoretically determined values for the isobaric ideal-gas heat capacity. For the siloxanes investigated in this work, the only ideal-gas isobaric heat capacity data available in the literature are those of Nannan and Colonna, ${ }^{33,34}$ which were determined by means of ab initio calculations in case of the linear siloxanes ${ }^{33}$ and low-pressure speed of sound measurements in case of $\mathrm{D}_{5} .{ }^{34}$ For the ab initio calculations, they state an uncertainty of less than $6 \%$ without any further information. Since no other data are available for comparison, a reliable statement on their accuracy is difficult. However, these data were published together with results for other linear and cyclic siloxanes, such as $\mathrm{MM}$ and $\mathrm{D}_{4}$. During the development of the fundamental equations of state for $\mathrm{MM}$ and $\mathrm{D}_{4}$, Thol et al. ${ }^{18,19}$ showed that uncertainties of approximately $3 \%$ are more likely when comparing to other literature data, which is well within the stated uncertainty of Nannan and Colonna. ${ }^{33}$ Because the method employed for MM and $\mathrm{D}_{4}$ was the same as for the higher-order siloxanes investigated in this work, a similar uncertainty of the data is expected here. Similar to our previous work, ${ }^{18-20}$ not only ideal-gas heat capacity data were used to adjust the parameters of the model but also data for the speed of sound and isobaric heat capacity beyond the ideal-gas limit. Furthermore, the temperature independent contribution $c_{p}^{\mathrm{o}}=4 R$ in the low-temperature limit and the asymptotic course of the $c_{p}^{\mathrm{o}}$ equation at very high temperatures were constrained. The results are shown in Figure 2.

The isobaric heat-capacity data of the ideal gas of the two linear siloxanes are represented within $0.3 \%$, except for one outlier of $\mathrm{MD}_{3} \mathrm{M}$ at $T=700 \mathrm{~K}$. The data of $\mathrm{D}_{5}$ are reproduced within $0.9 \%$ for temperatures $T \geq 400 \mathrm{~K}$. Data at lower temperatures deviate by up to $3.9 \%$, but fitting these values at low temperatures more accurately would negatively affect values pertaining the remaining temperature range and the speed of sound. Considering an estimated uncertainty of $3 \%$ to $6 \%$, the data might generally be overfitted and the expected uncertainty of isobaric heat-capacity data of the ideal gas calculated with the present equations of state is in the same range as the uncertainty of the underlying data. 


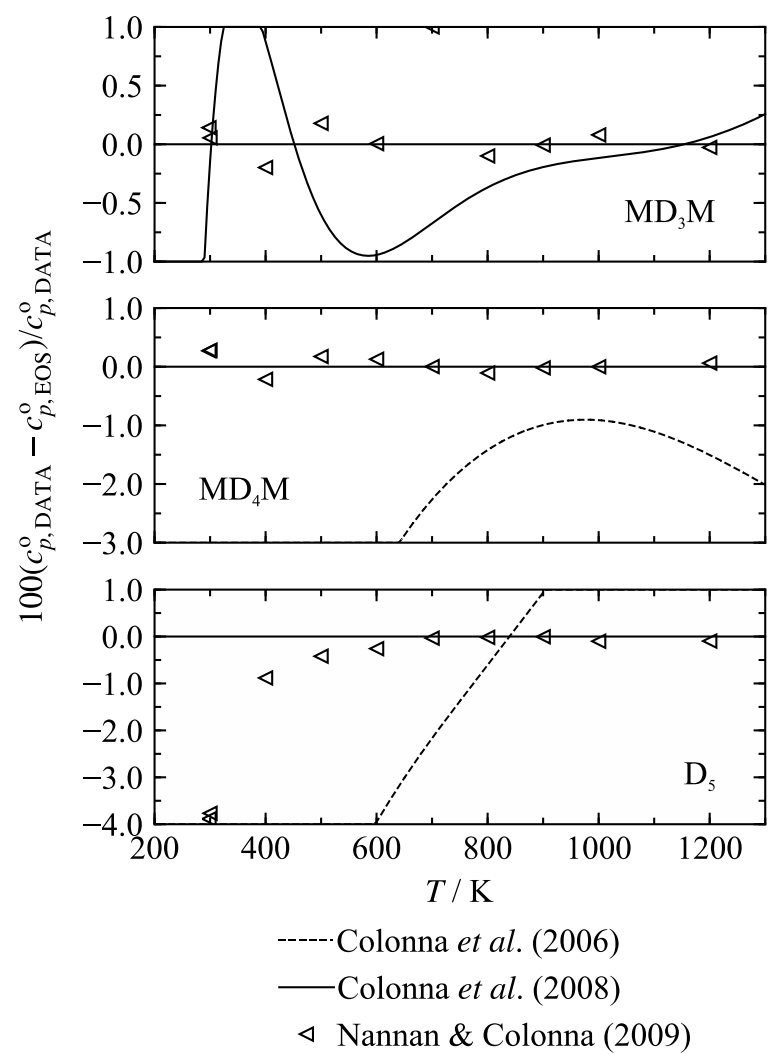

Figure 2. Percentage deviations of the isobaric heat-capacity data of the ideal gas of Nannan and Colonna ${ }^{33}$ from the present equations of state for $\mathrm{MD}_{3} \mathrm{M}, \mathrm{MD}_{4} \mathrm{M}$, and $\mathrm{D}_{5}$. Deviations with respect to the equations of state of Colonna et al. ${ }^{14,15}$ are shown for comparison.

The residual part consists of three types of alpha terms. The polynomial $\alpha_{\mathrm{Pol}}^{\mathrm{r}}$ and exponential $\alpha_{\operatorname{Exp}}^{\mathrm{r}}$ terms are sufficient to accurately represent thermodynamic properties in the entire fluid region except for the region in the vicinity of the critical point. For the description of this region, Gaussian bell-shaped ${ }^{35,36} \alpha_{\mathrm{GBS}}^{\mathrm{r}}$ and non-analytic terms ${ }^{24,37}$ were introduced. Non-analytic terms are needed in order to correctly model the divergence of the isochoric heat capacity and vanishing speed of sound at the critical point. However, the use of these terms requires a comprehensive and highly accurate dataset in the critical region, which is not available for the siloxanes investigated in this work. Furthermore, these terms may cause numerical problems when being applied to mixture models. Therefore, the functional form adopted here for $\mathrm{D}_{5}$, $\mathrm{MD}_{3} \mathrm{M}$, and $\mathrm{MD}_{4} \mathrm{M}$ is

$$
\begin{aligned}
\alpha^{\mathrm{r}}(\tau, \delta) & =\alpha_{\mathrm{Pol}}^{\mathrm{r}}(\tau, \delta)+\alpha_{\mathrm{Exp}}^{\mathrm{r}}(\tau, \delta)+\alpha_{\mathrm{GBS}}^{\mathrm{r}}(\tau, \delta) \\
& =\sum_{i=1}^{5} n_{i} \delta^{d_{i}} \tau^{t_{i}}+\sum_{i=6}^{10} n_{i} \delta^{d_{i}} \tau^{t_{i}} \exp \left(-\delta^{p_{i}}\right) \\
& +\sum_{i=11}^{15} n_{i} \delta^{d_{i}} \tau^{t_{i}} \exp \left[-\eta_{i}\left(\delta-\varepsilon_{i}\right)^{2}-\beta_{i}\left(\tau-\gamma_{i}\right)^{2}\right] .
\end{aligned}
$$

For all siloxanes, five terms of each type were used and the corresponding parameters are listed in Tables 3 to 5. Test values for computer implementation are provided in Table 6. The parameters were determined with the help of a non-linear fitting algorithm provided by the National 
Institute of Standards and Technology. ${ }^{38,39}$ In addition to experimental measurements, this algorithm allows for the application of various constraints ensuring the correct and consistent thermodynamic behavior of fluids also in regions where experimental data are not available. Further information is provided in Refs. ${ }^{38,40-43}$

Table 3. Parameters of the residual part of the present equation of state for dodecamethylpentasiloxane $\left(\mathrm{MD}_{3} \mathrm{M}\right)$, cf. Eq. (11).

\begin{tabular}{|c|c|c|c|c|c|c|c|c|}
\hline$i$ & $n_{i}$ & $t_{i}$ & $d_{i}$ & $p_{i}$ & $\eta_{i}$ & $\beta_{i}$ & $\gamma_{i}$ & $\varepsilon_{i}$ \\
\hline 1 & 0.040674325 & 1.000 & 4 & & & & & \\
\hline 2 & 4.4936509 & 0.370 & 1 & & & & & \\
\hline 3 & -6.0327468 & 0.718 & 1 & & & & & \\
\hline 4 & -1.0842396 & 0.790 & 2 & & & & & \\
\hline 5 & 0.65985153 & 0.590 & 3 & & & & & \\
\hline 6 & -2.3011802 & 2.380 & 1 & 2 & & & & \\
\hline 7 & -1.5022099 & 3.140 & 3 & 2 & & & & \\
\hline 8 & 0.5051725 & 0.620 & 2 & 1 & & & & \\
\hline 9 & -2.2363839 & 2.080 & 2 & 2 & & & & \\
\hline 10 & -0.071582853 & 1.042 & 7 & 1 & & & & \\
\hline 11 & 4.7053488 & 0.900 & 1 & & 1.043 & 0.860 & 1.357 & 0.725 \\
\hline 12 & -0.774783117 & 0.860 & 1 & & 20.00 & 1099 & 1.097 & 0.940 \\
\hline 13 & -0.68302991 & 2.060 & 3 & & 1.080 & 0.950 & 1.030 & 0.546 \\
\hline 14 & 0.41657104 & 0.550 & 2 & & 0.470 & 0.100 & 1.020 & 0.680 \\
\hline 15 & -1.1441135 & 0.690 & 2 & & 1.085 & 1.850 & 0.800 & 0.495 \\
\hline
\end{tabular}

Table 4. Parameters of the residual part of the present equation of state for tetradecamethylhexasiloxane (MD $\left.{ }_{4} \mathrm{M}\right)$, cf. Eq. (11).

\begin{tabular}{|c|c|c|c|c|c|c|c|c|}
\hline$i$ & $n_{i}$ & $t_{i}$ & $d_{i}$ & $p_{i}$ & $\eta_{i}$ & $\beta_{i}$ & $\gamma_{i}$ & $\varepsilon_{i}$ \\
\hline 1 & 0.053362183 & 1.000 & 4 & & & & & \\
\hline 2 & 2.8527871 & 0.300 & 1 & & & & & \\
\hline 3 & -3.8108356 & 0.680 & 1 & & & & & \\
\hline 4 & -0.95254215 & 0.913 & 2 & & & & & \\
\hline 5 & 0.44739021 & 0.434 & 3 & & & & & \\
\hline 6 & -2.5194015 & 2.330 & 1 & 2 & & & & \\
\hline 7 & -1.2945338 & 2.700 & 3 & 2 & & & & \\
\hline 8 & 0.43538523 & 0.610 & 2 & 1 & & & & \\
\hline 9 & -0.92015738 & 2.120 & 2 & 2 & & & & \\
\hline 10 & -0.054299195 & 1.121 & 7 & 1 & & & & \\
\hline 11 & 4.6112643 & 1.130 & 1 & & 0.810 & 0.526 & 1.340 & 0.977 \\
\hline 12 & -0.58630821 & 0.700 & 1 & & 17.30 & 700.0 & 1.108 & 0.920 \\
\hline 13 & -0.7391977 & 2.550 & 3 & & 0.892 & 0.720 & 1.190 & 0.650 \\
\hline 14 & -0.14001997 & 2.590 & 2 & & 0.820 & 0.056 & 1.680 & 1.060 \\
\hline 15 & -1.8085327 & 1.070 & 2 & & 0.847 & 1.300 & 0.860 & 0.659 \\
\hline
\end{tabular}


Table 5. Parameters of the residual part of the present equation of state for decamethylcyclopentasiloxane ( $\left.D_{5}\right)$, cf. Eq. (11).

\begin{tabular}{|c|c|c|c|c|c|c|c|c|}
\hline$i$ & $n_{i}$ & $t_{i}$ & $d_{i}$ & $p_{i}$ & $\eta_{i}$ & $\beta_{i}$ & $\gamma_{i}$ & $\varepsilon_{i}$ \\
\hline 1 & 0.0177345 & 1.000 & 4 & & & & & \\
\hline 2 & 4.3133088 & 0.430 & 1 & & & & & \\
\hline 3 & -6.1586863 & 0.754 & 1 & & & & & \\
\hline 4 & -1.4503945 & 0.840 & 2 & & & & & \\
\hline 5 & 0.9519342 & 0.720 & 3 & & & & & \\
\hline 6 & -2.3848036 & 2.350 & 1 & 2 & & & & \\
\hline 7 & -1.4114529 & 2.580 & 3 & 2 & & & & \\
\hline 8 & 0.7255071 & 0.660 & 2 & 1 & & & & \\
\hline 9 & -2.9966803 & 1.710 & 2 & 2 & & & & \\
\hline 10 & -0.0902228 & 1.0163 & 7 & 1 & & & & \\
\hline 11 & 6.3033323 & 1.114 & 1 & & 1.046 & 0.37 & 1.626 & 0.787 \\
\hline 12 & -1.0592923 & 1.850 & 3 & & 0.993 & 0.11 & 1.050 & 0.567 \\
\hline 13 & 0.79365281 & 0.900 & 2 & & 0.545 & 0.10 & 1.110 & 0.685 \\
\hline 14 & -1.8982515 & 1.050 & 2 & & 1.128 & 0.37 & 1.220 & 0.577 \\
\hline 15 & -0.01351964 & 1.090 & 1 & & 13.90 & 519 & 1.083 & 0.936 \\
\hline
\end{tabular}

Table 6. Test values for computer implementation. The number of digits does not refer to the accuracy of the data, but allows to numerically verify the correct implementation of the models.

\begin{tabular}{|c|c|c|c|c|c|c|}
\hline $\begin{array}{c}T \\
/ \mathrm{K} \\
\end{array}$ & $\begin{array}{c}\rho \\
/\left(\mathrm{mol} \cdot \mathrm{dm}^{-3}\right) \\
\end{array}$ & $\begin{array}{c}p \\
/ \mathrm{MPa} \\
\end{array}$ & $\begin{array}{c}h \\
/\left(\mathrm{J} \cdot \mathrm{mol}^{-1}\right) \\
\end{array}$ & $\begin{array}{c}s \\
/\left(\mathrm{J} \cdot \mathrm{mol}^{-1} \cdot \mathrm{K}^{-1}\right) \\
\end{array}$ & $\begin{array}{c}w \\
/\left(\mathrm{m} \cdot \mathrm{s}^{-1}\right)\end{array}$ & $\begin{array}{c}a \\
/\left(\mathrm{J} \cdot \mathrm{mol}^{-1}\right)\end{array}$ \\
\hline \multicolumn{7}{|c|}{$\mathbf{M D}_{3} \mathbf{M}$} \\
\hline 300 & 2.4 & 56.5643398 & -133761.828 & -403.543152 & 1241.26649 & -36267.3571 \\
\hline 390 & 0.0005 & 0.0016139843 & -31595.5295 & -48.7220551 & 92.0127172 & -15821.8965 \\
\hline 450 & 0.003 & 0.0110320958 & 7104.19531 & 27.6618505 & 97.5667091 & -9021.00267 \\
\hline 450 & 2.0 & 18.3032601 & -38524.8786 & -101.224710 & 728.435652 & -2125.38904 \\
\hline 600 & 2.0 & 70.6395352 & 100062.177 & 113.577309 & 902.133167 & -3403.97539 \\
\hline \multicolumn{7}{|c|}{$\mathrm{MD}_{4} \mathrm{M}$} \\
\hline 280 & 2.1 & 70.8719158 & -199382.667 & -595.997052 & 1346.73495 & -66252.0236 \\
\hline 420 & 0.0005 & 0.0017375886 & -46438.0435 & -75.4013910 & 87.2885442 & -18244.6365 \\
\hline 500 & 0.01 & 0.0391881825 & 17451.7844 & 38.3367562 & 90.1871428 & -5635.41188 \\
\hline 500 & 1.8 & 67.169626 & -10913.2743 & -99.7222037 & 982.279594 & 1631.36868 \\
\hline 650 & 1.5 & 31.6991170 & 127131.508 & 178.458722 & 637.354433 & -9999.40628 \\
\hline \multicolumn{7}{|c|}{$\mathrm{D}_{5}$} \\
\hline 290 & 2.7 & 36.3487297 & -122272.731 & -359.629958 & 1151.09861 & -31442.5359 \\
\hline 390 & 0.001 & 0.0032226439 & -14185.4999 & -14.0834572 & 93.6614237 & -11915.5955 \\
\hline 450 & 0.01 & 0.0358844583 & 20404.0115 & 48.6842603 & 97.0959266 & -5092.35152 \\
\hline 450 & 2.5 & 77.0798056 & -4880.23864 & -81.6230026 & 1044.97883 & 1018.19028 \\
\hline 650 & 1.8 & 14.8882334 & 129408.704 & 215.447596 & 415.207142 & -18903.4744 \\
\hline
\end{tabular}


Table 7. Fluid-specific thermodynamic properties of $\mathrm{MD}_{3} \mathrm{M}, \mathrm{MD}_{4} \mathrm{M}$, and $\mathrm{D}_{5}$. If no reference is given, the property was calculated in this work. Ad hoc critical-point measurements of siloxanes are not reported in the literature and are extremely difficult to obtain. The extrapolated critical temperatures reported by Flaningam ${ }^{44}$ $\left(T_{\mathrm{c}, \mathrm{MD} 3 \mathrm{M}}=627.61 \mathrm{~K}, T_{\mathrm{c}, \mathrm{MD} 4 \mathrm{M}}=653.16 \mathrm{~K}\right.$, and $\left.T_{\mathrm{c}, \mathrm{D} 5}=619.11 \mathrm{~K}\right)$ were used as initial values for the fit and slightly adjusted so that the equations of state optimally match with the underlying experimental data.

\begin{tabular}{|c|c|c|c|c|c|c|c|}
\hline Property & Unit & $\mathrm{MD}_{3} \mathrm{M}$ & Ref. & $\mathrm{MD}_{4} \mathrm{M}$ & Ref. & $\mathrm{D}_{5}$ & Ref. \\
\hline Critical temperature $T_{\mathrm{c}}$ & $\mathrm{K}$ & 628 & & 653.2 & 44 & 618.3 & \\
\hline Critical density $\rho_{\mathrm{c}}$ & $\mathrm{mol} \cdot \mathrm{dm}^{-3}$ & 0.7 & & 0.57 & & 0.81 & \\
\hline Critical pressure $p_{\mathrm{c}}$ & $\mathrm{kPa}$ & 953.95 & & 828.56 & & 1077.7 & \\
\hline $\begin{array}{l}\text { Normal-boiling point tempera- } \\
\text { ture } T_{\mathrm{B}}\end{array}$ & $\mathrm{K}$ & 503.02 & & 532.85 & & 484.10 & \\
\hline Triple-point temperature $T_{\mathrm{tr}}$ & $\mathrm{K}$ & 192 & 45 & 214.15 & 45 & 224.65 & 46 \\
\hline $\begin{array}{l}\text { Liquid triple-point density at } \\
\text { saturation } \rho_{\text {tr,liq. }}\end{array}$ & $\mathrm{mol} \cdot \mathrm{dm}^{-3}$ & 2.533 & & 2.111 & & 2.790 & \\
\hline Molar mass $M$ & $\mathrm{~g} \cdot \mathrm{mol}^{-1}$ & 384.839 & 47 & 458.9933 & 47 & 370.7697 & 47 \\
\hline
\end{tabular}

Several thermodynamic properties of special interest are listed in Table 7.

The computational time required to obtain vapor-liquid equilibrium properties can be greatly reduced if initial values for the calculations are computed with so-called ancillary equations. Such equations for the fluids under scrutiny are

$$
\begin{gathered}
\ln \left(\frac{p_{\mathrm{v}}}{p_{\mathrm{c}}}\right)=\left(\frac{T_{\mathrm{c}}}{T}\right) \sum_{i=1}^{5} n_{i}\left(1-\frac{T}{T_{\mathrm{c}}}\right)^{t_{i}}, \\
\frac{\rho^{\prime}}{\rho_{\mathrm{c}}}=1+\sum_{i=1}^{5} n_{i}\left(1-\frac{T}{T_{\mathrm{c}}}\right)^{t_{i}}, \\
\ln \left(\frac{\rho^{\prime \prime}}{\rho_{\mathrm{c}}}\right)=\sum_{i=1}^{6} n_{i}\left(1-\frac{T}{T_{\mathrm{c}}}\right)^{t_{i}} .
\end{gathered}
$$

Since these equations are used to reduce the calculation time of saturation properties from the fundamental equations of state, the parameters are not adjusted to experimental data but to the corresponding equations of state. In this way, starting values for the iterative determination of the vapor pressure and saturation densities can be provided very close to the correct solution. The parameters are listed in Table 8 and deviations from the values obtained with the Helmholtz-based thermodynamic models are illustrated in Figure 3.

\begin{tabular}{|c|c|c|c|c|c|c|}
\hline \multirow[b]{2}{*}{$i$} & \multicolumn{2}{|c|}{$\begin{array}{l}\text { Vapor pressure, } \\
\text { Eq. (12) }\end{array}$} & \multicolumn{2}{|c|}{$\begin{array}{c}\text { Saturated liquid density, } \\
\text { Eq. (13) }\end{array}$} & \multicolumn{2}{|c|}{$\begin{array}{c}\text { Saturated vapor density, } \\
\text { Eq. (14) }\end{array}$} \\
\hline & $n_{i}$ & $t_{i}$ & $n_{i}$ & $t_{i}$ & $n_{i}$ & $t_{i}$ \\
\hline & & & $\mathbf{M D}_{3} \mathbf{M}$ & & & \\
\hline 1 & -9.6774 & 1 & 3.326 & 0.42 & -4.0084 & 0.441 \\
\hline 2 & 3.973 & 1.5 & 3.889 & 1.46 & -7.913 & 1.244 \\
\hline 3 & -3.701 & 1.83 & -2.363 & 0.9 & -79.392 & 5.88 \\
\hline 4 & -9.1232 & 3.54 & -2.709 & 2.15 & -28.572 & 3.03 \\
\hline
\end{tabular}

Table 8. Parameters of the ancillary equations for vapor pressure, saturated liquid density, and saturated vapor density for $\mathrm{MD}_{3} \mathrm{M}, \mathrm{MD}_{4} \mathrm{M}$, and $\mathrm{D}_{5}$. 


\begin{tabular}{|c|c|c|c|c|c|c|}
\hline \multirow[b]{2}{*}{$i$} & \multicolumn{2}{|c|}{$\begin{array}{l}\text { Vapor pressure, } \\
\text { Eq. (12) }\end{array}$} & \multicolumn{2}{|c|}{$\begin{array}{c}\text { Saturated liquid density, } \\
\text { Eq. (13) }\end{array}$} & \multicolumn{2}{|c|}{$\begin{array}{c}\text { Saturated vapor density, } \\
\text { Eq. (14) }\end{array}$} \\
\hline & $n_{i}$ & $t_{i}$ & $n_{i}$ & $t_{i}$ & $n_{i}$ & $t_{i}$ \\
\hline 5 & -5.467 & 11.9 & 1.325 & 3.15 & -211.86 & 12.7 \\
\hline 6 & & & & & -1800 & 32 \\
\hline \multicolumn{7}{|c|}{ MD4M } \\
\hline 1 & -9.6745 & 1.0 & -0.034315 & 0.14 & -3.316 & 0.432 \\
\hline 2 & 1.3742 & 1.5 & 4.0324 & 0.45 & -8.324 & 1.085 \\
\hline 3 & 0.71467 & 2.2 & -2.7980 & 0.84 & -184.27 & 3.78 \\
\hline 4 & -12.967 & 3.2 & 2.2450 & 1.28 & 350.11 & 4.42 \\
\hline 5 & -7.201 & 11.4 & 0.41085 & 7.92 & -287.08 & 5.1 \\
\hline 6 & & & & & -267.25 & 13.0 \\
\hline \multicolumn{7}{|c|}{$D_{5}$} \\
\hline 1 & -9.256 & 1 & 1.0938 & 0.25 & -0.916 & 0.23 \\
\hline 2 & 3.987 & 1.5 & 5.254 & 0.79 & -5.911 & 0.68 \\
\hline 3 & -11.02 & 2.24 & -12.31 & 1.33 & -18.617 & 2.24 \\
\hline 4 & -19.286 & 3.48 & 19.364 & 1.9 & -74.29 & 5.1 \\
\hline 5 & 16.524 & 2.86 & -15.81 & 2.52 & -154.4 & 10.7 \\
\hline 6 & -8.14 & 11.6 & 5.983 & 3.22 & -284.1 & 18.9 \\
\hline
\end{tabular}
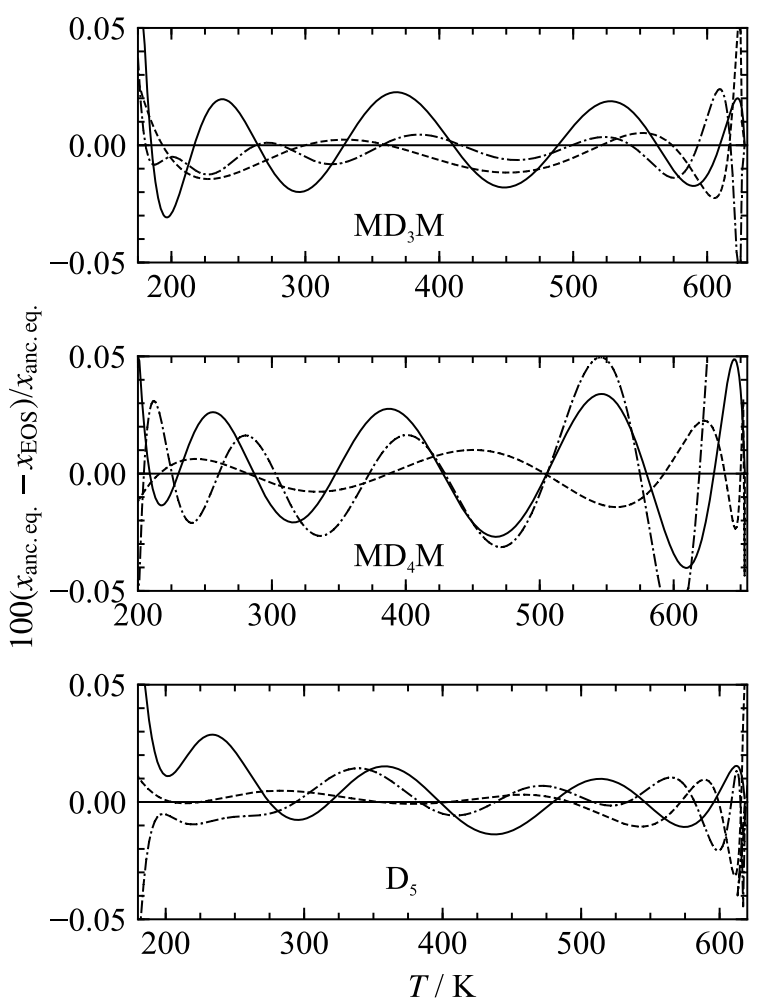

Figure 3. Percentage deviations of the ancillary equations for vapor pressure and saturation densities from the present equations of state for $\mathrm{MD}_{3} \mathrm{M}, \mathrm{MD}_{4} \mathrm{M}$, and $\mathrm{D}_{5}$. The solid curve represents the vapor pressure, whereas the dashed and dashed-dotted curves illustrate the saturated liquid and vapor densities, respectively. 


\section{COMPARISON WITH LITERATURE DATA}

In order to evaluate the capability of the obtained equations of state, values computed with the models were compared with experimental data reported in the literature in terms of relative deviations according to

$$
\Delta X=100 \frac{x_{\mathrm{DATA}}-x_{\mathrm{EOS}}}{x_{\mathrm{DATA}}},
$$

where $x$ is an arbitrary thermodynamic property. In addition to a graphical analysis, a statistical evaluation was carried out by means of the average absolute relative deviation (AAD)

$$
\mathrm{AAD}=\frac{1}{N} \sum_{i=1}^{N}\left|\Delta X_{i}\right|
$$

where $N$ is the number of data points of the individual datasets. It is common practice to report the AAD depending on the phase or the temperature/density ranges. However, since the database of measured properties is rather limited, only an overall AAD for each dataset is given in Table 9.

Table 9. Average absolute relative deviations (AAD / \%) of experimental data calculated with the present equations

\begin{tabular}{|c|c|c|c|c|c|c|}
\hline Author & Year & Fluid & $N$ & $T_{\min }-T_{\max } / \mathrm{K}$ & $p_{\min }-p_{\max } / \mathrm{MPa}$ & $\mathrm{AAD} / \%$ \\
\hline \multicolumn{7}{|c|}{ Isobaric heat capacity of the ideal gas } \\
\hline \multirow[t]{3}{*}{ Nannan \& Colonna ${ }^{33}$} & 2009 & $\mathrm{MD}_{3} \mathrm{M}$ & 10 & $298-1200$ & & 0.30 \\
\hline & & $\mathrm{MD}_{4} \mathrm{M}$ & 10 & $298-1200$ & & 0.12 \\
\hline & & $\mathrm{D}_{5}$ & 10 & $298-1200$ & & 0.95 \\
\hline \multicolumn{7}{|l|}{ Vapor pressure } \\
\hline Abbas et al..$^{17}$ & 2011 & $\mathrm{D}_{5}$ & 19 & $329-484$ & & 2.6 \\
\hline Fischer \& Weiss ${ }^{48}$ & 1986 & $\mathrm{D}_{5}$ & 1 & 489.96 & & 1.3 \\
\hline \multirow[t]{3}{*}{ Flaningam $^{44}$} & 1986 & $\mathrm{MD}_{3} \mathrm{M}$ & 15 & $395-516$ & & 0.051 \\
\hline & & $\mathrm{MD}_{4} \mathrm{M}$ & 11 & $449-546$ & & 0.22 \\
\hline & & $\mathrm{D}_{5}$ & 8 & $383-497$ & & 0.18 \\
\hline Hurd $^{49}$ & 1946 & $\mathrm{D}_{5}$ & 1 & 483.01 & & 2.5 \\
\hline \multirow[t]{2}{*}{ Lei et al..$^{50}$} & 2010 & $\mathrm{MD}_{4} \mathrm{M}$ & 1 & 298.15 & & 8.8 \\
\hline & & $\mathrm{D}_{5}$ & 1 & 298.15 & & 5.4 \\
\hline Mills \& MacKenzie ${ }^{51}$ & 1954 & $\mathrm{D}_{5}$ & 1 & 375.12 & & 3.0 \\
\hline $\begin{array}{l}\text { Palczewska-Tulinska \& } \\
\text { Oracz }^{52}\end{array}$ & 2005 & $\mathrm{D}_{5}$ & 23 & $348-480$ & & 8.9 \\
\hline \multirow[t]{2}{*}{ Stull ${ }^{53}$} & 1947 & $\mathrm{MD}_{3} \mathrm{M}$ & 10 & $329-494$ & & 21.0 \\
\hline & & $\mathrm{MD}_{4} \mathrm{M}$ & 10 & $346-519$ & & 35.0 \\
\hline Tanaka $^{54}$ & 1960 & $\mathrm{D}_{5}$ & 1 & 353.12 & & 16.0 \\
\hline Thompson $^{55}$ & 1953 & $\mathrm{MD}_{3} \mathrm{M}$ & 1 & 501.66 & & 3.8 \\
\hline \multirow[t]{3}{*}{ Waterman et al..$^{56}$} & 1958 & $\mathrm{MD}_{3} \mathrm{M}$ & 1 & 501.46 & & 1.4 \\
\hline & & $\mathrm{MD}_{4} \mathrm{M}$ & 1 & 414.53 & & 4.4 \\
\hline & & $\mathrm{D}_{5}$ & 1 & 484.36 & & 0.21 \\
\hline
\end{tabular}
of state, where $N$ is the number of data points. 


\begin{tabular}{|c|c|c|c|c|c|c|}
\hline Author & Year & Fluid & $N$ & $T_{\min }-T_{\max } / \mathrm{K}$ & $p_{\min }-p_{\max } / \mathrm{MPa}$ & $\mathrm{AAD} / \%$ \\
\hline \multirow[t]{2}{*}{ Wilcock $^{57}$} & 1946 & $\mathrm{MD}_{3} \mathrm{M}$ & 2 & $390-503$ & & 1.5 \\
\hline & & $\mathrm{D}_{5}$ & 2 & $374-484$ & & 2.0 \\
\hline \multicolumn{7}{|l|}{ Density } \\
\hline \multirow[t]{3}{*}{ This work } & 2019 & $\mathrm{MD}_{3} \mathrm{M}$ & 50 & $273-358$ & $<91$ & 0.053 \\
\hline & & $\mathrm{MD}_{4} \mathrm{M}$ & 50 & $273-358$ & $<91$ & 0.025 \\
\hline & & $\mathrm{D}_{5}$ & 50 & $273-358$ & $<91$ & 0.038 \\
\hline Fischer \& Weiss ${ }^{48}$ & 1986 & $\mathrm{D}_{5}$ & 1 & 298.19 & 0.1 & 0.093 \\
\hline \multirow[t]{2}{*}{ Fox et al..$^{58}$} & 1947 & $\mathrm{MD}_{3} \mathrm{M}$ & 1 & 298.14 & 0.101325 & 0.63 \\
\hline & & $\mathrm{MD}_{4} \mathrm{M}$ & 1 & 293.14 & 0.101325 & 0.062 \\
\hline Golik \& Cholpan ${ }^{59}$ & 1961 & $\mathrm{MD}_{3} \mathrm{M}$ & 1 & 303.13 & 0.101325 & 0.29 \\
\hline \multirow[t]{2}{*}{ Hunter et al. ${ }^{60}$} & 1946 & $\mathrm{MD}_{3} \mathrm{M}$ & 1 & 298.14 & 0.101325 & 0.12 \\
\hline & & $\mathrm{MD}_{4} \mathrm{M}$ & 1 & 298.14 & 0.101325 & 0.065 \\
\hline \multirow[t]{3}{*}{ Hurd $^{49}$} & 1946 & $\mathrm{MD}_{3} \mathrm{M}$ & 5 & $272-354$ & 0.101325 & 0.059 \\
\hline & & $\mathrm{MD}_{4} \mathrm{M}$ & 5 & $273-354$ & 0.101325 & 0.071 \\
\hline & & $\mathrm{D}_{5}$ & 5 & $273-354$ & 0.101325 & 0.072 \\
\hline $\begin{array}{l}\text { McLure \& Barbarin-Cas- } \\
\text { tillo }^{61}\end{array}$ & 1994 & $\mathrm{D}_{5}$ & 15 & $302-452$ & 0.101325 & 0.085 \\
\hline \multirow[t]{2}{*}{ McLure et al. ${ }^{62}$} & 1977 & $\mathrm{MD}_{3} \mathrm{M}$ & 16 & $297-409$ & 0.101325 & 0.019 \\
\hline & & $\mathrm{MD}_{4} \mathrm{M}$ & 19 & $293-412$ & 0.101325 & 0.11 \\
\hline Mills \& MacKenzie ${ }^{51}$ & 1954 & $\mathrm{D}_{5}$ & 2 & $293-302$ & 0.101325 & 0.063 \\
\hline $\begin{array}{l}\text { Palczewska-Tulinska \& } \\
\text { Oracz }^{52}\end{array}$ & 2005 & $\mathrm{D}_{5}$ & 23 & $295-454$ & 0.101325 & 0.21 \\
\hline Patnode \& Wilcock ${ }^{63}$ & 1946 & $\mathrm{D}_{5}$ & 1 & 293.14 & 0.101325 & 0.018 \\
\hline \multirow[t]{2}{*}{ Povey et al..$^{64}$} & 2003 & $\mathrm{MD}_{3} \mathrm{M}$ & 1 & 239.15 & 0.101325 & 0.022 \\
\hline & & $\mathrm{MD}_{4} \mathrm{M}$ & 1 & 239.15 & 0.101325 & 0.51 \\
\hline Schedemann ${ }^{65}$ & 2013 & $\mathrm{D}_{5}$ & 460 & $287-437$ & $<130$ & 0.036 \\
\hline Tanaka $^{54}$ & 1960 & $\mathrm{D}_{5}$ & 1 & 293.14 & 0.101325 & 0.049 \\
\hline \multirow[t]{3}{*}{ Waterman et al. ${ }^{56}$} & 1958 & $\mathrm{MD}_{3} \mathrm{M}$ & 1 & 293.14 & 0.101325 & 0.002 \\
\hline & & $\mathrm{MD}_{4} \mathrm{M}$ & 1 & 293.14 & 0.101325 & 0.15 \\
\hline & & $\mathrm{D}_{5}$ & 1 & 293.14 & 0.101325 & 0.055 \\
\hline \multirow[t]{2}{*}{ Weissler ${ }^{66}$} & 1949 & $\mathrm{MD}_{3} \mathrm{M}$ & 1 & 303.13 & 0.101325 & 0.002 \\
\hline & & $\mathrm{MD}_{4} \mathrm{M}$ & 1 & 303.13 & 0.101325 & 0.61 \\
\hline \multicolumn{7}{|l|}{ Speed of sound } \\
\hline \multirow[t]{3}{*}{ This work } & 2019 & $\mathrm{MD}_{3} \mathrm{M}$ & 74 & $221-500$ & $<125$ & 0.10 \\
\hline & & $\mathrm{MD}_{4} \mathrm{M}$ & 57 & $299-500$ & $<126$ & 0.039 \\
\hline & & $\mathrm{D}_{5}$ & 64 & $249-500$ & $<125$ & 0.12 \\
\hline Golik \& Cholpan ${ }^{59}$ & 1961 & $\mathrm{MD}_{3} \mathrm{M}$ & 1 & 303.13 & 0.101325 & 0.020 \\
\hline Nannan et al..$^{34}$ & 2007 & $\mathrm{D}_{5}$ & 40 & $485-511$ & $<1$ & 0.19 \\
\hline \multirow[t]{2}{*}{ Povey et al..$^{64}$} & 2003 & $\mathrm{MD}_{3} \mathrm{M}$ & 1 & 293.15 & 0.101325 & 0.23 \\
\hline & & $\mathrm{MD}_{4} \mathrm{M}$ & 1 & 293.15 & 0.101325 & 0.26 \\
\hline \multirow[t]{3}{*}{ Waterman et al. ${ }^{56}$} & 1958 & $\mathrm{MD}_{3} \mathrm{M}$ & 1 & 293.14 & 0.101325 & 0.15 \\
\hline & & $\mathrm{MD}_{4} \mathrm{M}$ & 1 & 293.14 & 0.101325 & 0.096 \\
\hline & & $\mathrm{D}_{5}$ & 1 & 293.14 & 0.101325 & 0.044 \\
\hline \multirow[t]{2}{*}{ Weissler ${ }^{66}$} & 1949 & $\mathrm{MD}_{3} \mathrm{M}$ & 2 & $303-324$ & 0.101325 & 0.18 \\
\hline & & $\mathrm{MD}_{4} \mathrm{M}$ & 2 & $303-324$ & 0.101325 & 0.35 \\
\hline
\end{tabular}




\begin{tabular}{lcccccc} 
Author & Year & Fluid & $N$ & $T_{\min }-T_{\max } / \mathrm{K}$ & $p_{\min }-p_{\max } / \mathrm{MPa}$ & $\mathrm{AAD} / \%$ \\
\hline Isobaric heat capacity & & & & & & \\
Abbas et al. ${ }^{17}$ & 2011 & $\mathrm{D}_{5}$ & 35 & $273-444$ & 0.101325 & 0.39 \\
McLure $^{67}$ & 1977 & $\mathrm{MD}_{3} \mathrm{M}$ & 2 & $303-323$ & 0.101325 & 4.9 \\
& & $\mathrm{MD}_{4} \mathrm{M}$ & 2 & $303-324$ & 0.101325 & 3.1 \\
Palczewska-Tulinska \& & \multirow{2}{*}{2005} & $\mathrm{D}_{5}$ & 35 & $288-474$ & 0.101325 & 0.89 \\
\hline Orac5 & & &
\end{tabular}

\subsection{Vapor Pressure}

The available vapor-pressure measurements for the three investigated siloxanes are listed in Table 9, and percentage deviations of the data from values calculated with the present equations of state are shown in Figure 4 . For the linear siloxanes, only Flaningam ${ }^{44}$ reported measurements at several temperatures, while other authors provide one single vapor pressure measurement. The data of Stull ${ }^{53}$ are part of a comprehensive collection of literature data for several hundred fluids and were derived from correlations. Comparisons with experimental data and equations of state for other siloxanes ${ }^{18-20}$ showed huge deviations of the corresponding vaporpressure data reported by Stull. ${ }^{53}$ Therefore, these data were not considered during the development of the present equations, but listed for completeness only. Average absolute relative deviations of more than $20 \%$ confirm this decision. Therefore, only the data of Flaningam ${ }^{44}$ were used for fitting the vapor-pressure curve. The uncertainties of the temperature and pressure measurements reported by Flaningam ${ }^{44}$ yield a combined expanded uncertainty $(k=2)$ of up to $0.29 \%$ for the highest pressure. However, the sample purity of $99 \%$ was not considered for the calculation of the uncertainties because no information about the impurities was given. To verify his results, Flaningam ${ }^{44}$ carried out test measurements with water and two other fluids, but no numerical values are provided in the publication. He states an average vapor pressure error of $0.07 \%$. According to his calculation ("average pressure error = $\sum\left(\left(p_{\text {expl }}-p_{\text {calcd }}\right) / p_{\text {calcd }} \times 100\right) / N$ "), Flaningam ${ }^{44}$ did not consider different algebraic signs (positive and negative deviations cancel out each other), which falsifies this value. Therefore, no statement on the uncertainty can be made here. Comparisons of his measurements of hexamethyldisiloxane (MM), which were released in the same publication, with accurate vapor pressure data of Scott et al. ${ }^{68}$ show deviations of approximately $0.5 \%$. Therefore, the uncertainty of the vapor pressure obtained with the equations of state of $\mathrm{MD}_{3} \mathrm{M}, \mathrm{MD}_{4} \mathrm{M}$, and $\mathrm{D}_{5}$ are expected to be in the same range. Figure 4 shows that all data are reproduced within $0.34 \%$, which is well within that estimated uncertainty range. For $\mathrm{D}_{5}$, this estimate is confirmed by the vapor-pressure data point of Waterman et al., ${ }^{56}$ which deviates by $-0.2 \%$ from the value calculated with the thermodynamic model and from the measurement reported by Flaningam. ${ }^{44}$ In contrast, deviations of $1.4 \%\left(\mathrm{MD}_{3} \mathrm{M}\right)$ and $4.4 \%\left(\mathrm{MD}_{4} \mathrm{M}\right)$ are observed for the linear siloxanes. In case of $\mathrm{D}_{5}$, 
there is an additional comprehensive dataset provided by Abbas et al. ${ }^{17}$ They carried out a comprehensive study on the thermophysical properties of siloxanes including vapor-pressure measurements on $\mathrm{MM}, \mathrm{MDM}, \mathrm{MD}_{2} \mathrm{M}, \mathrm{D}_{4}$, and $\mathrm{D}_{5}$. However, as already discussed in previous publications, ${ }^{18-20}$ it is assumed that their measurements by means of a comparative ebulliometer were carried out inadequately. Systematic deviations of approximately $-2 \%$ from values calculated with the present equation of state and measurements reported by Flaningam ${ }^{44}$ can be observed. Moreover, extensive scatter of more than 5\% is present, which is in line with the observations made in previous works. ${ }^{18-20}$ The same holds for the measurements of PalczewskaTulińska and Oracz. ${ }^{52}$ As already found for $\mathrm{D}_{4},{ }^{19}$ their data deviate by up to $12 \%$ from the present equation of state and the data of Flaningam. ${ }^{44}$

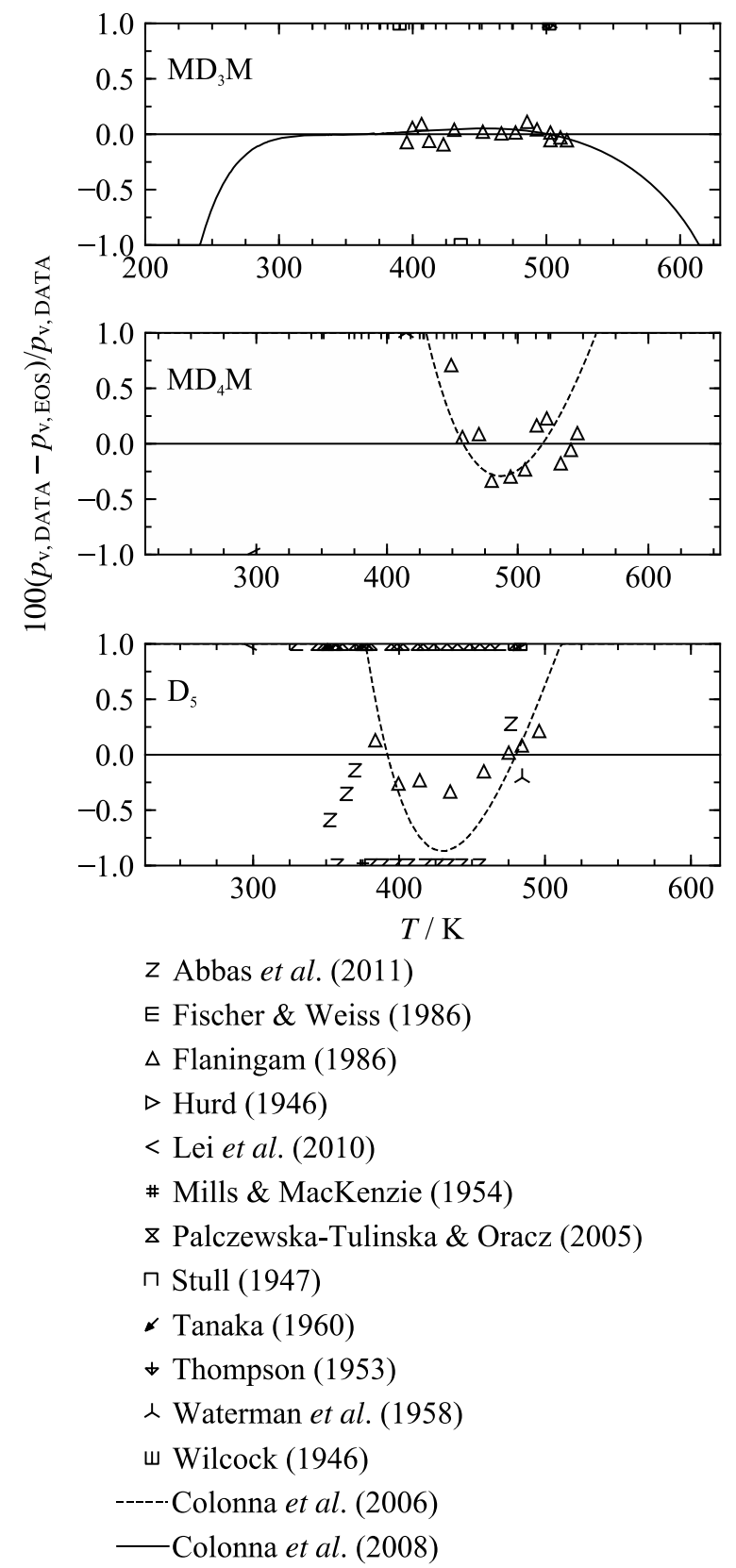

Figure 4. Percentage deviations of experimental vapor-pressure data from values calculated with the present equations of state for $\mathrm{MD}_{3} \mathrm{M}, \mathrm{MD}_{4} \mathrm{M}$, and $\mathrm{D}_{5}$. 
Based on this analysis, the expected uncertainty related to vapor pressure calculated with the present equations of state is $0.5 \%$ in the temperature region in which experimental data are available, as shown in Figure 4.

\subsection{Density}

In section 2, new density measurements for the three investigated siloxanes are presented for temperatures between $273 \mathrm{~K}$ and $358 \mathrm{~K}$ with a maximum pressure of $91 \mathrm{MPa}$, which were the basic data for the development of the present equations of state. Experimental data from the literature are listed in Table 9. The high-pressure measurements for $\mathrm{MD}_{3} \mathrm{M}$ and $\mathrm{MD}_{4} \mathrm{M}$ reported here are the only liquid density data at elevated pressures. Figures 5 and 6 show that the data are represented with the present equations of state within $0.1 \%$, except for one state point of $\mathrm{MD}_{3} \mathrm{M}$. Most of the data deviate by less than $0.05 \% \quad\left(\mathrm{AAD}_{\mathrm{MD} 3 \mathrm{M}}=0.053 \%\right.$, $\mathrm{AAD}_{\mathrm{MD} 4 \mathrm{M}}=0.025 \%$ ) from the equations. Since these data were not available to Colonna $e t$ al. ${ }^{14,15}$ deviations from their thermodynamic models amount up to $1.2 \%\left(\mathrm{MD}_{3} \mathrm{M}\right)$ and $1.8 \%$ $\left(\mathrm{MD}_{4} \mathrm{M}\right)$, particularly at elevated pressures.

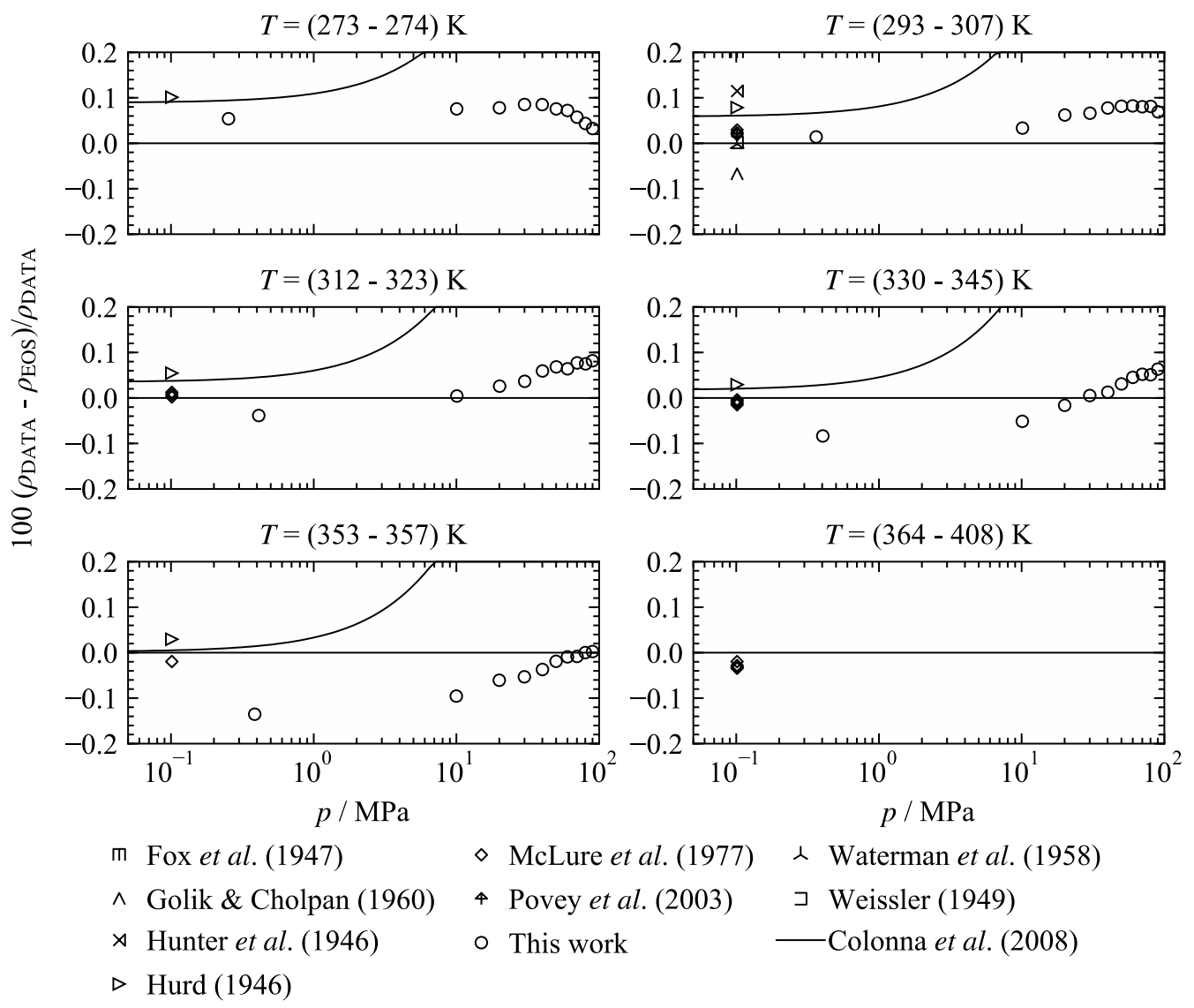

Figure 5. Percentage deviations of experimental density data from values calculated with the present equation of state for $\mathrm{MD}_{3} \mathrm{M}$. 
For $\mathrm{D}_{5}$, Schedemann ${ }^{65}$ carried out a comprehensive investigation on density, comprising 460 state points in a temperature range from $287 \mathrm{~K}$ to $437 \mathrm{~K}$ and pressures of up to $130 \mathrm{MPa}$. Measurements were performed with an Anton Paar vibrating tube densimeter, but no information was provided about experimental uncertainties. However, since it is the same apparatus as the one later applied by Abbas et al. ${ }^{17}$ to investigate other siloxanes, a similar uncertainty of $0.1 \%$ to $0.15 \%$ in the data can be assumed. For further information, see Thol et al. ${ }^{20}$ As illustrated in Figures 7 and 8 , the present equation of state represents the data well within this uncertainty $(\mathrm{AAD}=0.036 \%)$. Only 13 out of 460 state points deviate by more than $0.1 \%$, where the maximum deviation is $0.16 \%$. The measurements carried out in the present work (experimental uncertainty of $0.11 \%$ ) cover temperatures between the isotherms of Schedemann. ${ }^{65}$ Only the highest isotherm was measured at the same temperature $(357 \mathrm{~K})$ in order to directly compare both datasets. The datasets exhibit a systematic offset of approximately $0.05 \%$ from each other, which is well within the experimental uncertainties of both measurements. The good agreement is shown in Figure 7. All of our measurements are represented within $0.1 \%$ (AAD $=0.038 \%$ ).

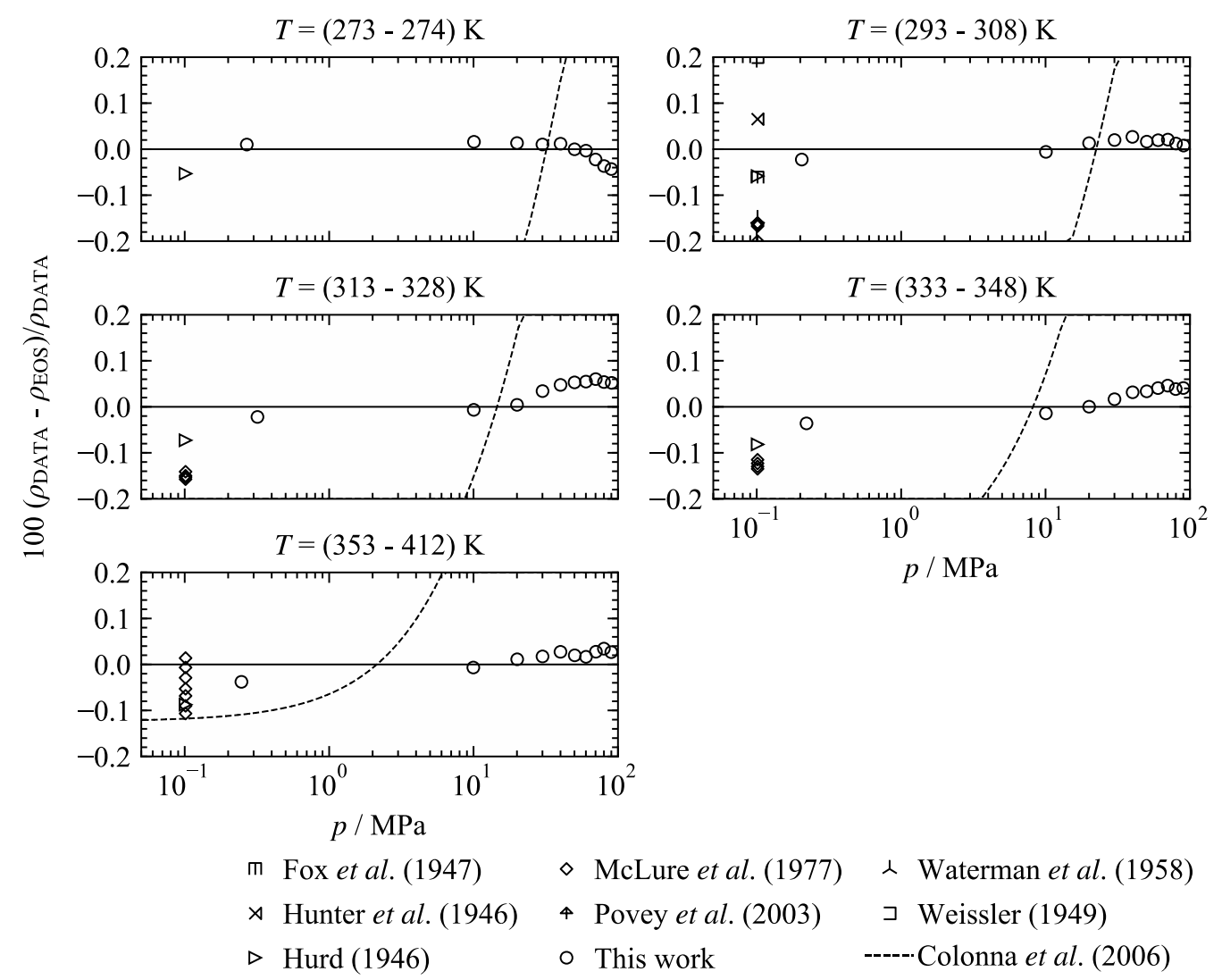

Figure 6. Percentage deviations of experimental density data from values calculated with the present equation of state for $\mathrm{MD}_{4} \mathrm{M}$. 

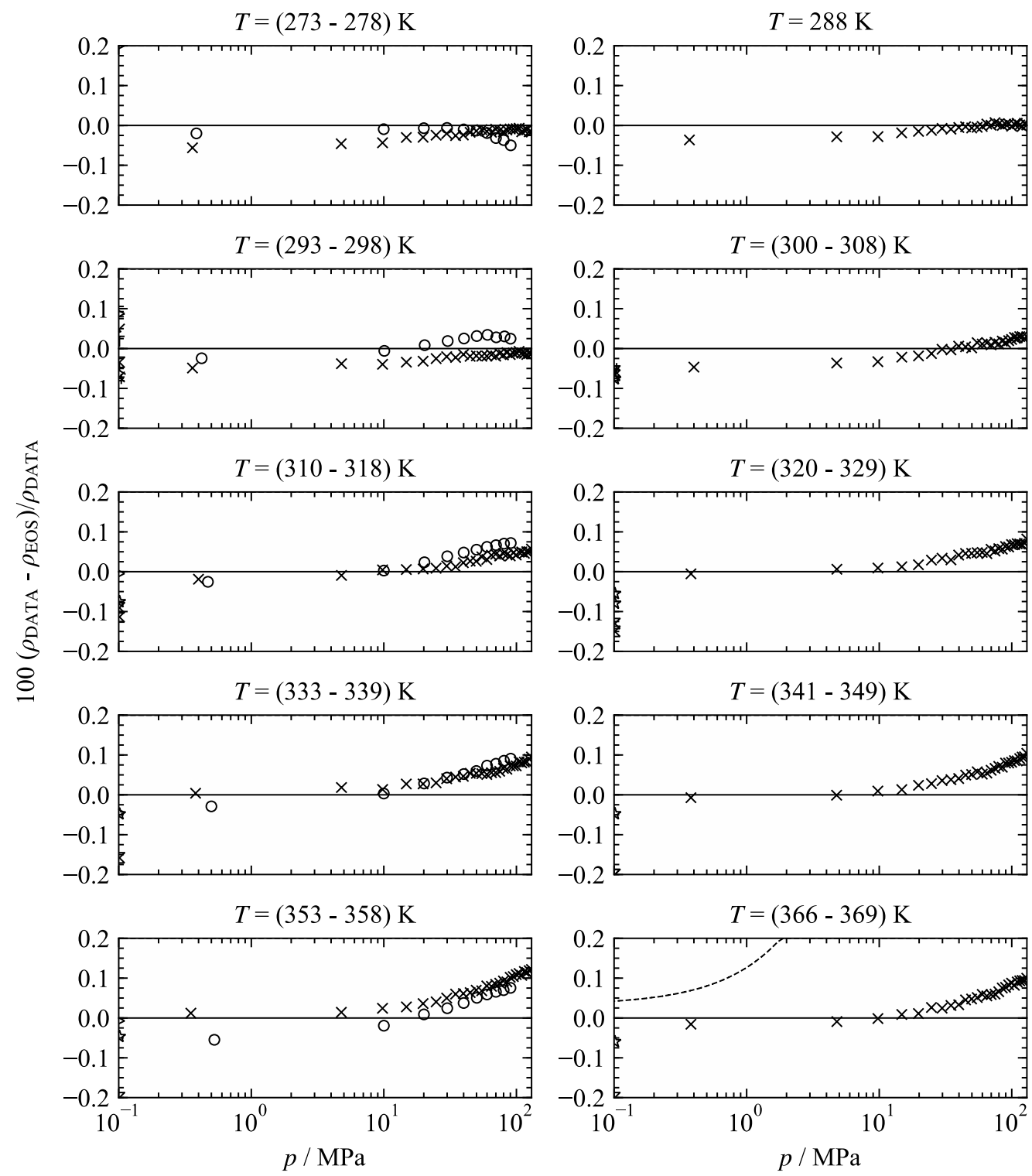

E Fischer \& Weiss (1986)

× Palczewska-Tulinska \& Oracz (2005) ○ This work

$\triangleright$ Hurd (1946)

T Patnode \& Wilcock (1946)

ᄉ Waterman et al. (1958)

* McLure \& Barbarin-Castillo (1994)

$\times$ Schedemann (2013)

----.Colonna et al. (2006)

\# Mills \& MacKenzie (1954)

- Tanaka (1960)

Figure 7. Percentage deviations of experimental density data from values calculated with the present equation of state for $\mathrm{D}_{5}$ for temperatures $T<370 \mathrm{~K}$. 

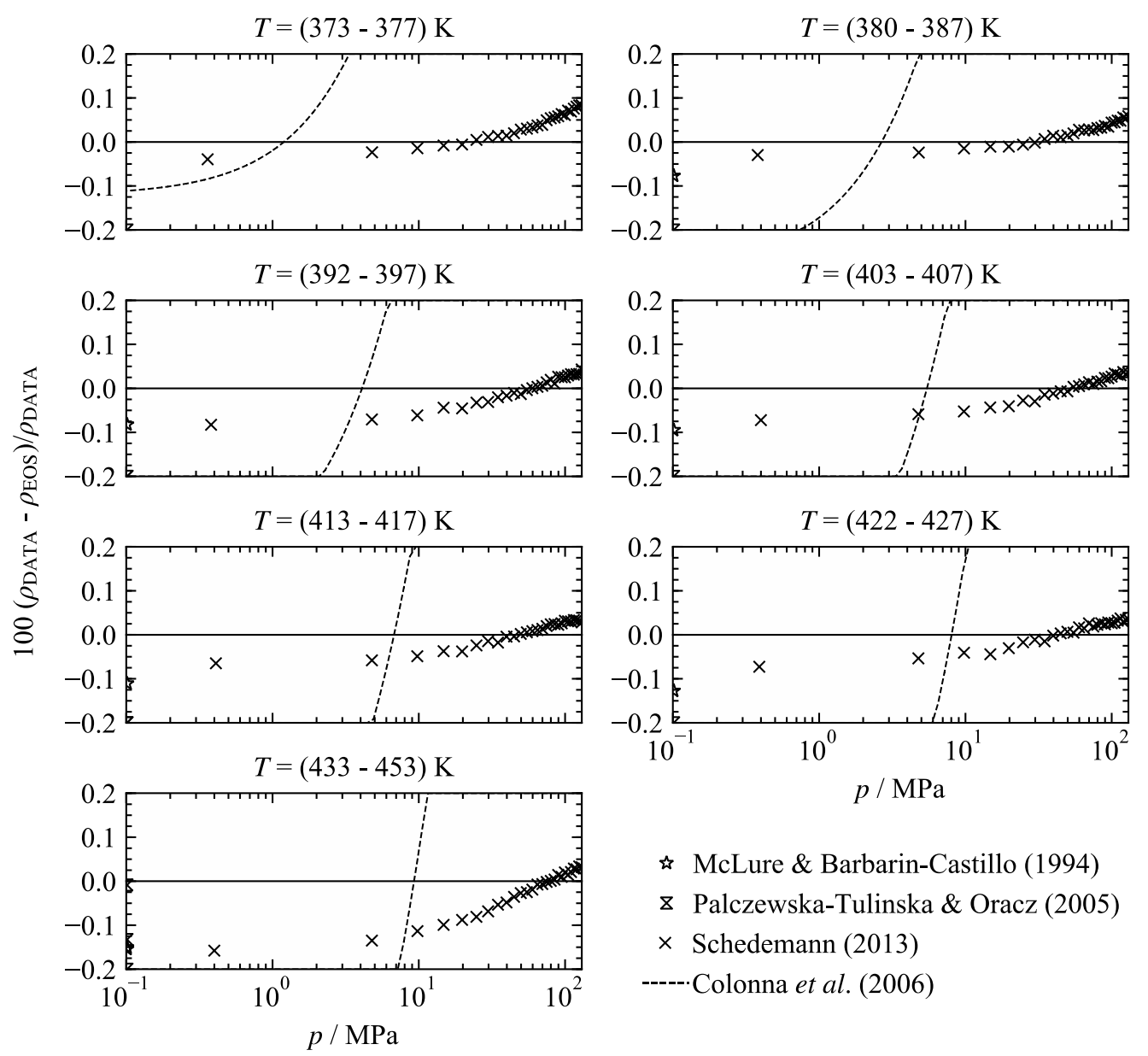

\& McLure \& Barbarin-Castillo (1994)

× Palczewska-Tulinska \& Oracz (2005)

$\times$ Schedemann (2013)

-----Colonna et al. (2006)

Figure 8. Percentage deviations of experimental density data from values calculated with the present equation of state for $\mathrm{D}_{5}$ for temperatures $T>370 \mathrm{~K}$.

In Figure 9, percentage deviations of density data measured at atmospheric pressure from the present equations of state are depicted. Especially for the linear siloxanes $\mathrm{MD}_{3} \mathrm{M}$ and $\mathrm{MD}_{4} \mathrm{M}$, only a few datasets with more than two state points are available. Data at atmospheric pressure were not included in the fitting process. Instead, low pressure data measured in this work and provided by Schedemann ${ }^{65}$ were applied and the atmospheric data were taken for comparison only. For $\mathrm{MD}_{3} \mathrm{M}$, all data except for the single state point of Fox et al. ${ }^{58}$ agree within $0.1 \%$ and are reproduced by the present equation of state in the same range. The data of McLure et $_{\text {al. }}{ }^{62}$ deviate by less than $0.04 \%(\mathrm{AAD}=0.019 \%)$, whereas the data of Hurd ${ }^{49}$ exhibit slightly higher deviations of up to $0.1 \%(\mathrm{AAD}=0.059 \%)$. In the case of $\mathrm{MD}_{4} \mathrm{M}$, the data of $\operatorname{Hurd}^{49}(\mathrm{AAD}=0.071 \%)$ are represented with a similar quality as for $\mathrm{MD}_{3} \mathrm{M}$, whereas the data of McLure et al. ${ }^{62}$ exhibit a systematic negative offset of up to $-0.17 \%$ (AAD $=0.11 \%$ ). Since the data of Hurd ${ }^{49}$ match with the low-pressure data measured in this work, this behavior is assumed to be correct. 

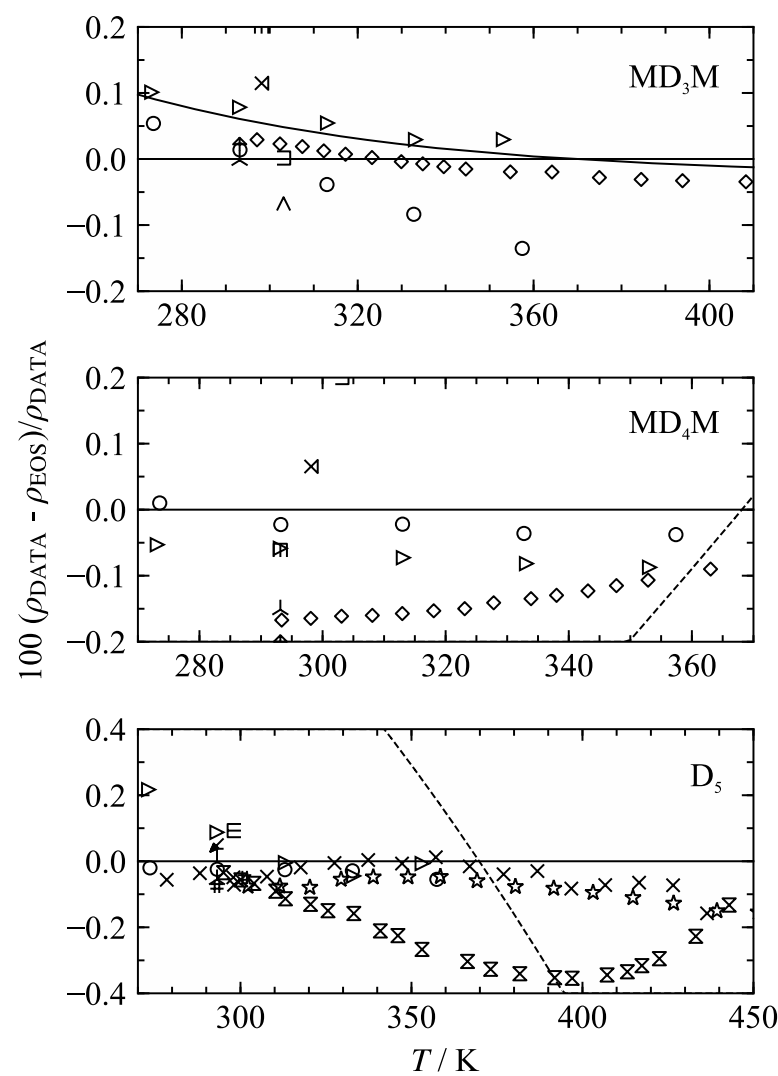

E Fischer \& Weiss (1986)

m Fox et al. (1947)

$\wedge$ Golik \& Cholpan (1960)

$\rtimes$ Hunter et al. (1946)

$\triangleright$ Hurd (1946)

* McLure \& Barbarin-Castillo (1994)

$\diamond$ McLure et al. (1977)

* Mills \& MacKenzie (1954)

z Palczewska-Tulinska \& Oracz (2005)

T Patnode \& Wilcock (1946)

4 Povey et al. (2003)

$\times$ Schedemann (2013)

* Tanaka (1960)

○ This work

ᄉ Waterman et al. (1958)

$\sqsupset$ Weissler (1949)

-----Colonna et al. (2006)

- Colonna et al. (2008)

Figure 9. Percentage deviations of experimental density data at atmospheric pressure from values calculated with the present equations of state for $\mathrm{MD}_{3} \mathrm{M}, \mathrm{MD}_{4} \mathrm{M}$, and $\mathrm{D}_{5}$. The pressure of the data from this work and by Schedemann ${ }^{65}$ were measured at pressures slightly above $1 \mathrm{~atm}$, but are nonetheless included in this comparison.

For $\mathrm{D}_{5}$, there are five different datasets available comprising more than two state points. The data measured in this work and provided by Schedemann ${ }^{65}$ again agree within their experimental uncertainty and are represented within $0.08 \%$, except for one state point of Schedemann ${ }^{65}$ at the highest temperature $T=436.44 \mathrm{~K}$. Measurements of McLure and BarbarinCastillo $^{61}$ agree with these two datasets and are, thus, reproduced within $0.15 \%$ 
$(\mathrm{AAD}=0.085 \%)$. The data of Hurd ${ }^{49}$ deviate by less than $0.09 \%$, except for one outlier at the lowest temperature $T=273.15 \mathrm{~K}$. Only the data of Palczewska-Tulińska and Oracz ${ }^{52}$ $(\mathrm{AAD}=0.21 \%)$ exhibit a different trend when compared to other measurements and the present equation of state. This is in agreement with the analysis of their data for $\mathrm{D}_{4}$, where the same course in terms of density deviations could be observed. ${ }^{19}$ Therefore, these data are assumed to be less accurate than the other measurements.

Based on this analysis, the expected uncertainty of calculated density data for the three investigated siloxanes are expected to be $0.15 \%$ in the liquid region up to $360 \mathrm{~K}$. For $\mathrm{D}_{5}$, uncertainties increase up to $0.2 \%$ between $360 \mathrm{~K}$ and $440 \mathrm{~K}$. 


\subsection{Speed of Sound}

Figures 10 to 12 illustrate percentage deviations of experimental speed of sound data from values calculated with the present equation of state. The equations of state of Colonna et al. ${ }^{14,15}$ are not shown because they deviate by up to $15 \%$ from the present data. For the two linear siloxanes, data are available only for the liquid state. The measurements carried out in this work are the only datasets measured at elevated pressure. Therefore, they were the basic data for the development of the present equations of state. Most of the data are reproduced within the experimental uncertainty specified in section 3. However, this uncertainty does not include the sample purity, which may have a significant impact as was already observed for other siloxanes. ${ }^{18-20} \mathrm{~A}$ few single measurements are available at atmospheric pressure, but no information about the quality of the data was given. However, all of the data agree within the expected uncertainty of the calculated data of $0.2 \%$.
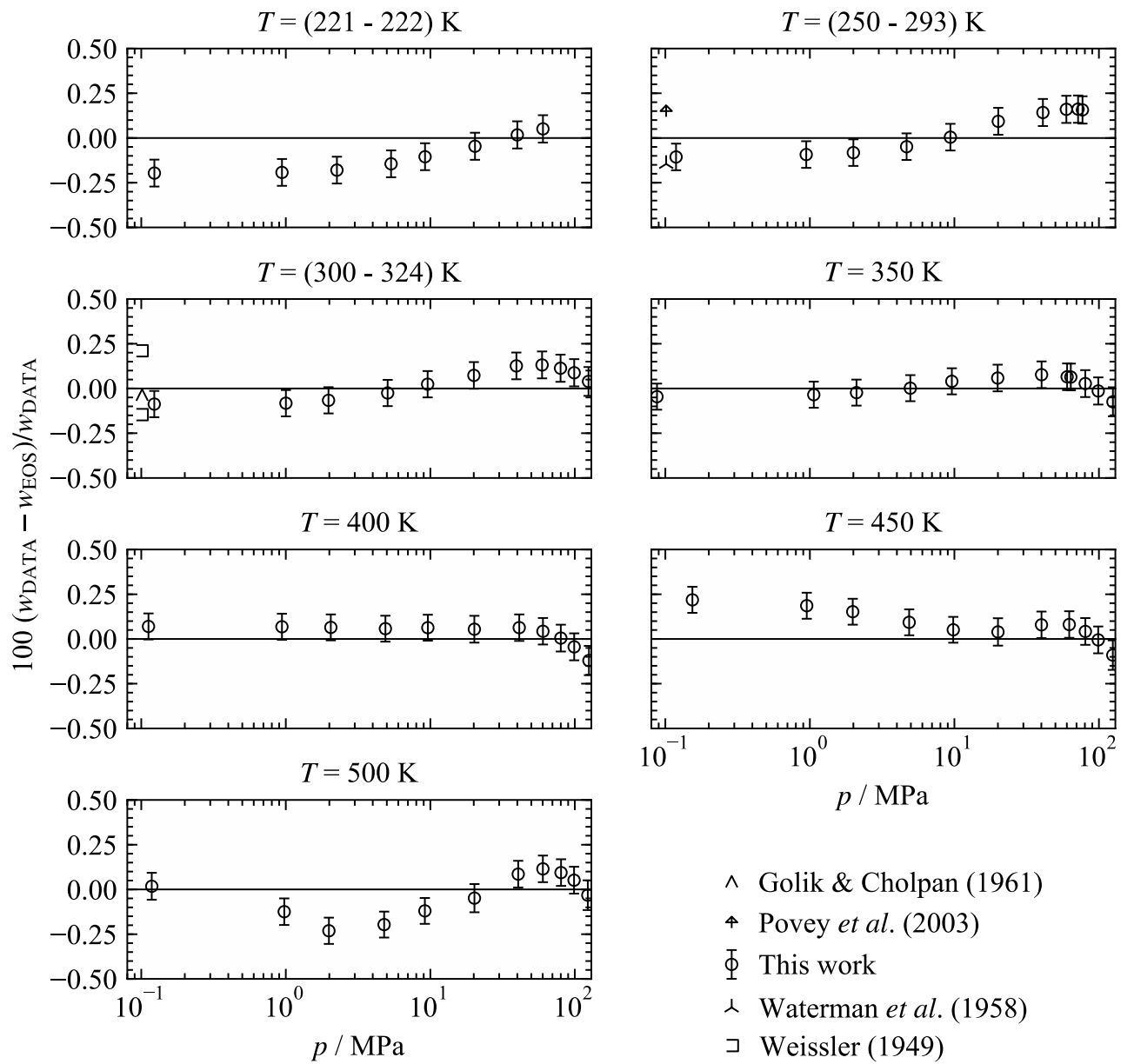

$\wedge$ Golik \& Cholpan (1961)

4 Povey et al. (2003)

$\Phi$ This work

^ Waterman et al. (1958)

$\sqsupset$ Weissler (1949)

Figure 10. Percentage deviations of experimental speed of sound data from values calculated with the present equation of state for $\mathrm{MD}_{3} \mathrm{M}$. The experimental uncertainty of the data measured in this work is shown with error bars. Uncertainties related to the sample purity were not considered because no information is available on the impurities, cf. section 3 . The equation of Colonna et al. ${ }^{15}$ is not shown because the corresponding deviations exceed the scale of the plots. 

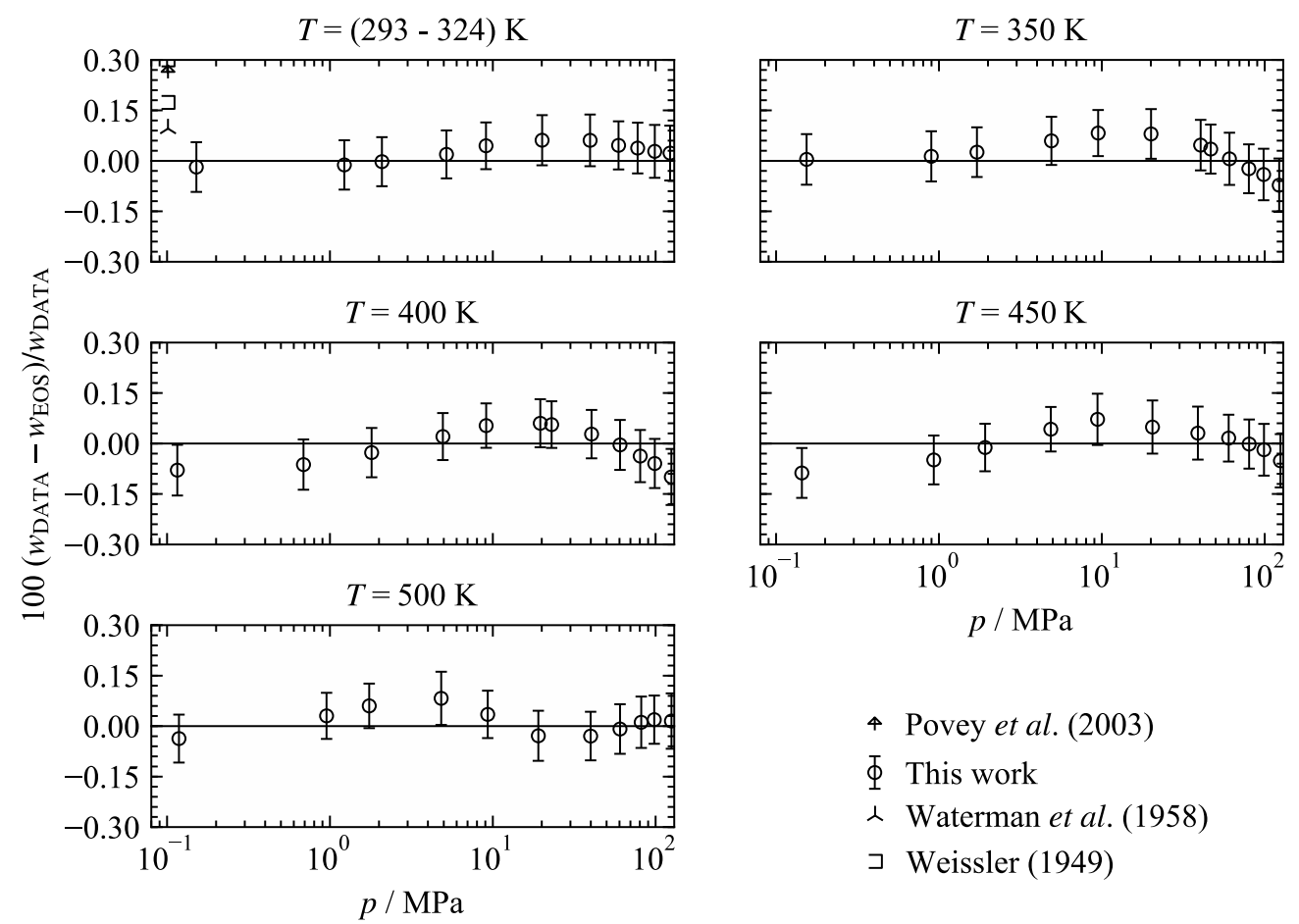

4 Povey et al. (2003)

$\Phi$ This work

\ Waterman et al. (1958)

$\sqsupset$ Weissler (1949)

Figure 11. Percentage deviations of experimental speed of sound data from values calculated with the present equation of state for $\mathrm{MD}_{4} \mathrm{M}$. The experimental uncertainty of the data measured in this work is shown with error bars. Uncertainties related to the sample purity were not considered because no information is available on the impurities, cf. section 3. The equation of Colonna et al. ${ }^{14}$ is not shown because the corresponding deviations exceed the scale of the plots.

For $\mathrm{D}_{5}$, the representation of the liquid-phase data is similar to that for the linear siloxanes, except for the lowest isotherm. Again, most of the data are reproduced within the experimental uncertainty, which was determined without considering the sample purity. Measurements at $250 \mathrm{~K}$ systematically differ from the present equation by $0.6 \%$. It was not possible to fit these data better without deteriorating other properties, especially the density. Therefore, it is assumed that the problem is related to the data rather than the equation. However, since those data are the only ones available in the literature, they were nonetheless included here. The gaseous phase was investigated by Nannan et ll $^{34}$ in a restricted temperature range of $480 \mathrm{~K}$ to $510 \mathrm{~K}$ in the ideal-gas state. The data are reproduced within $0.35 \%$, except for three state points at $T=510 \mathrm{~K}$, which are outliers.

Based on the new measurements presented in this work, the uncertainty of speed of sound data of the liquid is expected to be $0.2 \%$ for all three siloxanes. No statement can be made for the gaseous phase of the linear siloxanes. Since the database for $\mathrm{D}_{5}$ is rather restricted in the gaseous state, the deviations from the data of Nannan et al. ${ }^{34}$ can only be treated as an indication for the accuracy of the present equation of state, but no reliable assessment is possible. 

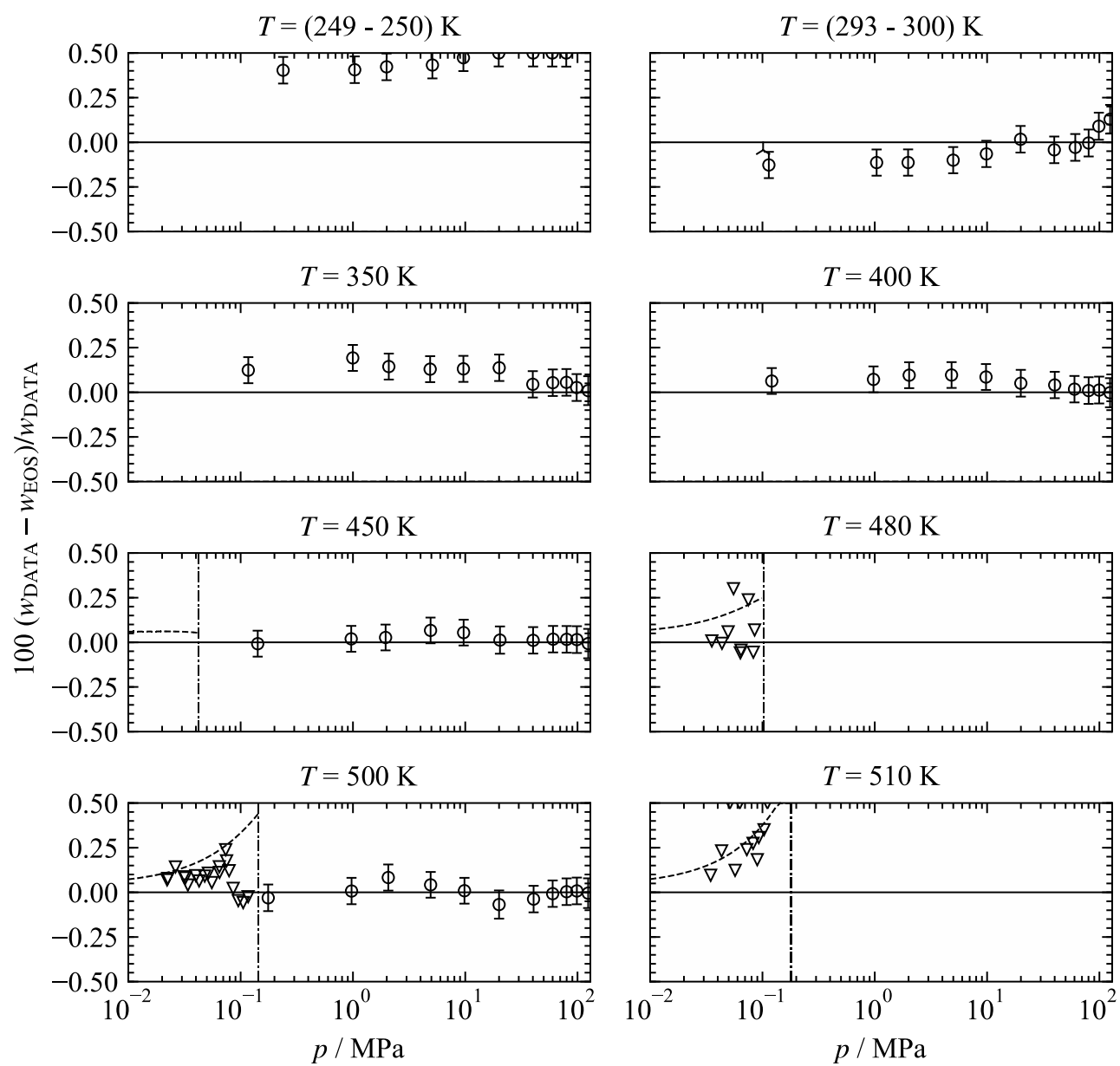

$\nabla$ Nannan et al. (2007)

^ Waterman et al. (1958) -----Phase boundary

$\Phi$ This work

Colonna et al. (2006)

Figure 12. Percentage deviations of experimental speed of sound data from values calculated with the present equation of state for $\mathrm{D}_{5}$. The experimental uncertainty of the data measured in this work is shown with error bars. Uncertainties related to the sample purity were not considered because no information is available on the impurities, cf. section 3 . The equation of Colonna et al. ${ }^{14}$ is not shown in the liquid phase because the corresponding deviations exceed the scale of the plots. 


\subsection{Isobaric Heat Capacity}

In comparison to the density and speed of sound, the database for the isobaric heat capacity is scarce, cf. Table 9. The two state points of McLure ${ }^{67}$ for both linear siloxanes were not measured, but numerically extrapolated. There is no information on the accuracy available and significant deviations of those data were already observed for other siloxanes. For $\mathrm{D}_{5}$, there are two contradictory datasets, those of Abbas et al. ${ }^{17}$ and Palczewska-Tulińska and Oracz. ${ }^{52}$ As explained in the publication of the equation of state for hexamethyldisiloxane, ${ }^{18}$ the main focus was given to the accurate representation of speed of sound data, and the less accurate isobaric heat-capacity data were fitted such that it does not interfere with other properties. The data of Abbas et al. ${ }^{17}$ are represented within the experimental uncertainty of $1 \%$, whereas the data of Palczewska-Tulińska and Oracz $^{52}$ deviate by up to $2 \%$. This is in line with the results observed for $\mathrm{D}_{4}$ and other siloxanes, where the data of Palczewska-Tulińska and Oracz ${ }^{52}$ significantly deviate from the equation and other literature data. Based on the data of Abbas et al., ${ }^{17}$ expected uncertainties of liquid isobaric heat-capacity values at atmospheric pressure calculated with the present equation of state are $1 \%$.

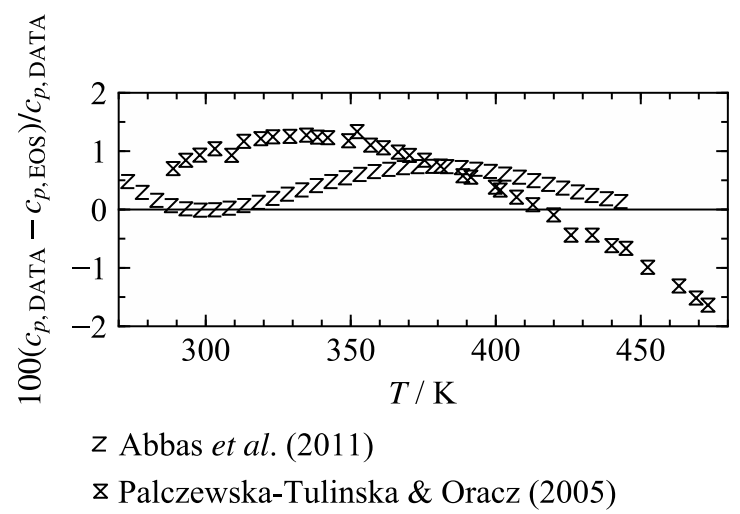

Figure 13. Percentage deviations of experimental heat-capacity data from values calculated with the present equation of state for $\mathrm{D}_{5}$. The equation of Colonna et al. ${ }^{14}$ is not shown because the corresponding deviations exceed the scale of the plot. 


\section{EXTRAPOLATION BEHAVIOR}

For the development of an equation of state, both correct physical and extrapolation behavior are equally important as the accurate representation of experimental data. Especially for fluids with a restricted experimental database, such as the siloxanes investigated in this work, the correct modeling of the physical behavior is essential for the application of the equations in regions and for properties where no experimental information is available. Furthermore, their implementation in mixture models requires a smooth extrapolation behavior to avoid numerical problems in regions outside the range of validity of the equation. The correct description of the thermal behavior ( $T-p-\rho$ relation) has been well known for decades, where special focus was given to the thermal virial coefficients, ideal curves, and extreme conditions of temperature, pressure, and density. Over the last 15 years, several thermodynamic quantities including caloric properties, e.g., isochoric heat capacity, speed of sound, phase identification parameter, ${ }^{69}$ or Grüneisen coefficient, ${ }^{70}$ were identified, which are useful for the assessment of the correct physical behavior. A comprehensive overview is presented, e.g., by Lemmon et al., ${ }^{38}$ Thol et al. ${ }^{41}$ or Herrig et al. ${ }^{71}$ In Figures 14 to 16 , only exemplary plots are shown although a full analysis of these properties was carried out for each siloxane investigated in this work. The course of each property follows the correct trend as described by the aforementioned authors. No unreasonable slopes, curvatures, or overhangs can be observed. 

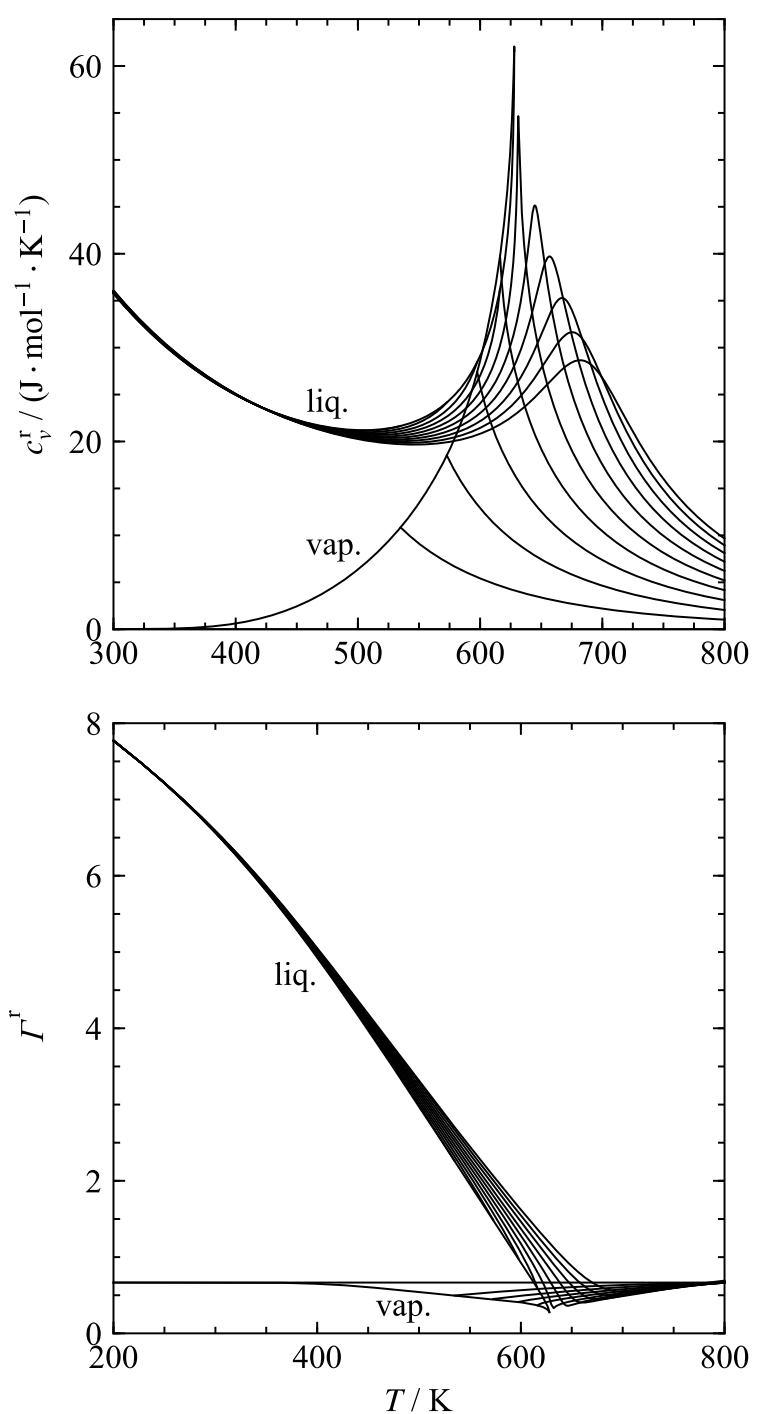

Figure 14. Residual isochoric heat capacity (top) and residual Grüneisen parameter (bottom) as a function of temperature along selected isobars for $\mathrm{MD}_{3} \mathrm{M}$. 

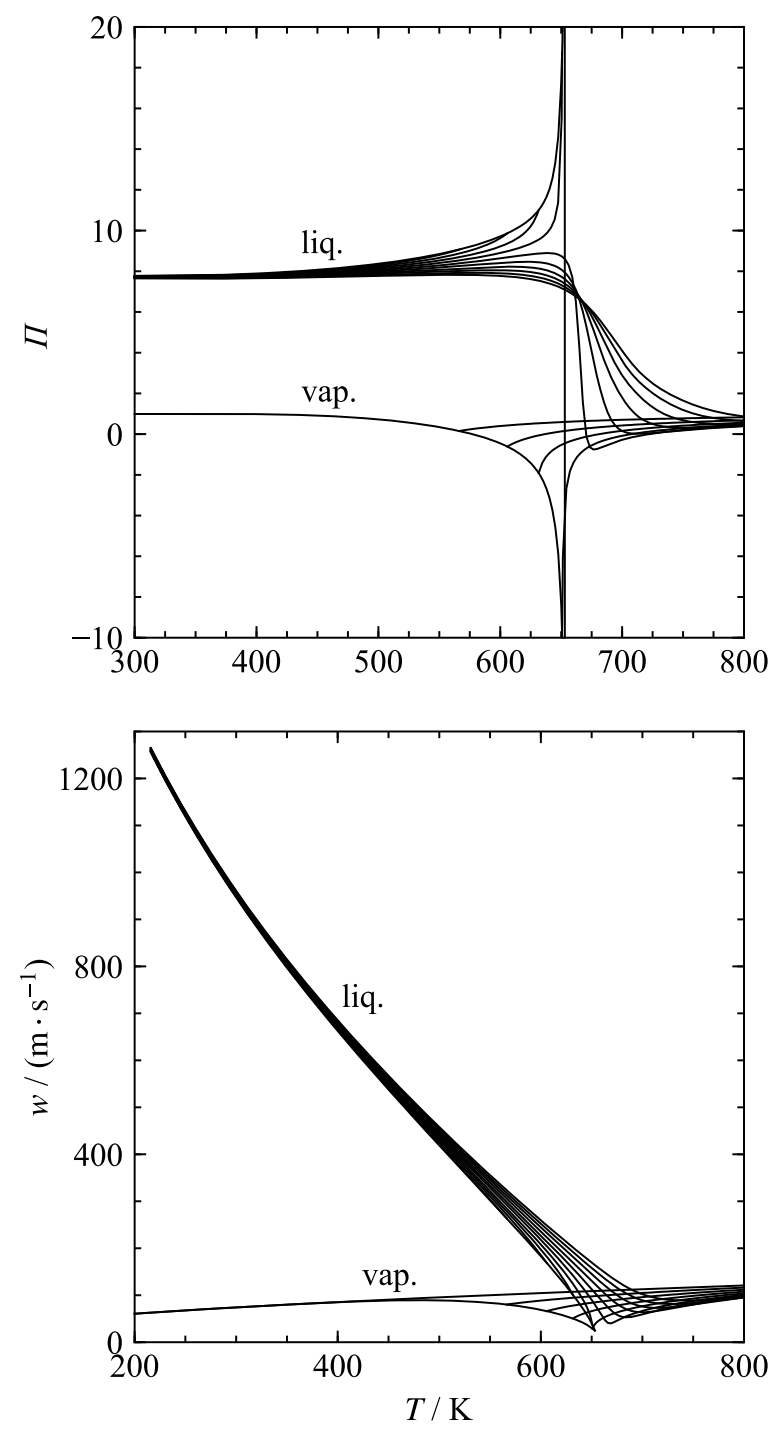

Figure 15. Phase identification parameter (top) and speed of sound (bottom) as a function of temperature along selected isobars for $\mathrm{MD}_{4} \mathrm{M}$. 

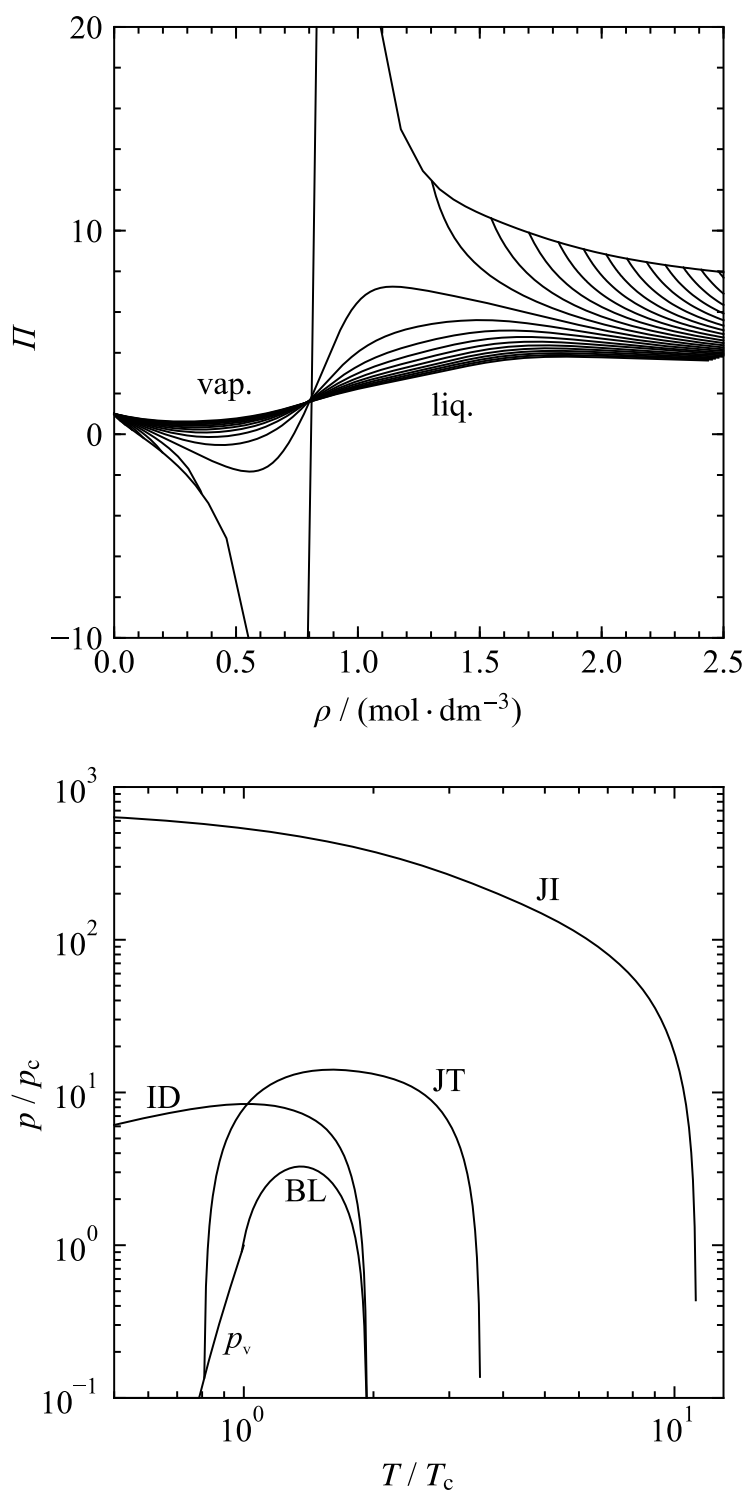

Figure 16. Phase identification parameter as a function of density along selected isotherms (top) and ideal curves ${ }^{72}$ (bottom) for $\mathrm{D}_{5}$. JI: Joule inversion curve, JT: Joule-Thomson inversion curve, ID: ideal curve, BL: Boyle curve, $p_{\mathrm{v}}$ : vapor-pressure curve. 


\section{ESTIMATION OF THE FUNDAMENTAL DERIVATIVE OF GAS DYNAMICS}

The so-called Bethe-Zel'dovich-Thompson (BZT) fluids are named after the scientists who first postulated them. ${ }^{5-7}$ For such fluids, theoretically, gas dynamics phenomena are inverted if compared to ideal gases if they occur in a limited thermodynamic region of dense vapor states, close to saturation, and the vapor-liquid critical point. For these fluids and in that thermodynamic region, a secondary thermodynamic property called the fundamental derivative of gas dynamics $^{7}$

$$
\Gamma_{\mathrm{GD}}=1+\frac{\rho}{w}\left(\frac{\partial w}{\partial \rho}\right)_{s}=\frac{v^{3}}{2 w^{2}}\left(\frac{\partial^{2} p}{\partial v^{2}}\right)_{s}
$$

is negative.

For the estimation of the fundamental derivative of gas dynamics, a reliable and accurate equation of state is necessary. One of the reasons why Colonna et al. ${ }^{14,15}$ developed fundamental equations in terms of the Helmholtz energy was the study of non-classical gas dynamics. However, these equations were parameterized to an experimental database with a very limited number of state points. As Eq. (17) shows, thermal and caloric properties are required for the calculation of the fundamental derivative of gas dynamics. Thus, for a reliable assessment of this property, a correct description of, e.g., the density and speed of sound is essential. In the present work, new equations of state were developed for three siloxanes, which are based on comprehensive measurements of density and speed of sound. Based on these equations, the fundamental derivative of gas dynamics was evaluated along the saturated vapor line as suggested by Colonna et al., ${ }^{8} \mathrm{cf}$. Figure 17. For $\mathrm{MD}_{3} \mathrm{M}$, the prediction of $\Gamma_{\mathrm{GD}}$ with both equations (Colonna et $a l .{ }^{15}$ and this work) agrees very well. In the case of the two other siloxanes, the absolute minimum is more distinct with the present equations than with the correlations of Colonna et $a l .{ }^{14,15}$ The fundamental derivative of gas dynamics is negative for all three siloxanes for saturated states close to the critical point. Therefore, these fluids could be used in experiments aimed at proving the existence of non-classical gas dynamics. Furthermore, Figure 18 shows that, for siloxanes belonging to the homologous series, the relation between the minimum value of $\Gamma_{\mathrm{GD}}$ along the saturated vapor line and the molar mass is approximately linear. As known from previous investigations, ${ }^{8}$ the more complex the molecule is (in case of homologous molecules), the lower is the value of $\Gamma_{\mathrm{GD}}$. Although no experimental data are available in the investigated temperature range, it is assumed that this behavior is indeed present because it is predicted from five entirely independent equations of state.

It can be expected that also in case of cyclic siloxanes a similar linear relation between the fundamental derivative of gas dynamics and the molecular weight exists. However, improved thermodynamic models are available only for $\mathrm{D}_{4}{ }^{19}$ and $\mathrm{D}_{5} . \Gamma_{\mathrm{GD}}$ is positive for all vapor states of $\mathrm{D}_{4}$, while for $\mathrm{D}_{5}$ a small region in which $\Gamma_{\mathrm{GD}}$ is negative is predicted by the model presented in this work. 

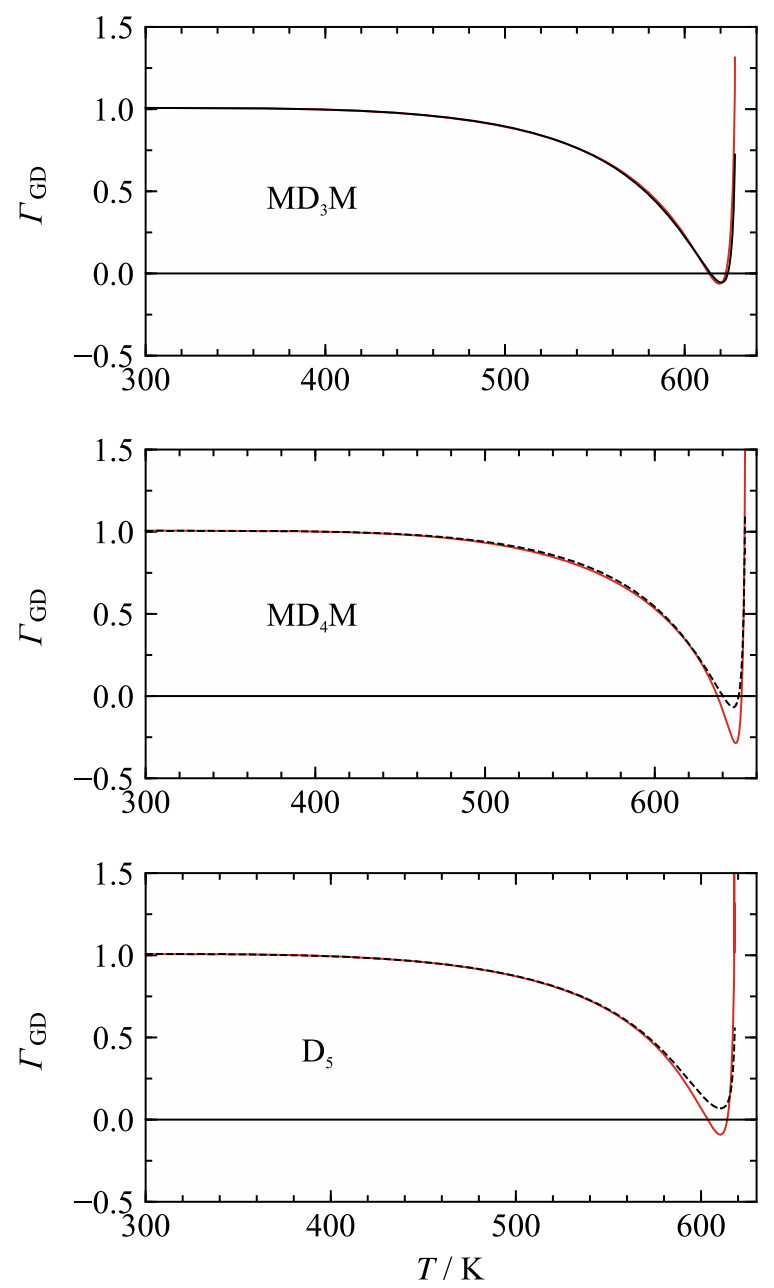

-----Colonna et al. (2006) - This work

-Colonna et al. (2008)

Figure 17. Fundamental derivative of gas dynamics as a function of temperature along the saturated vapor line of $\mathrm{MD}_{3} \mathrm{M}, \mathrm{MD}_{4} \mathrm{M}$, and $\mathrm{D}_{5}$. 

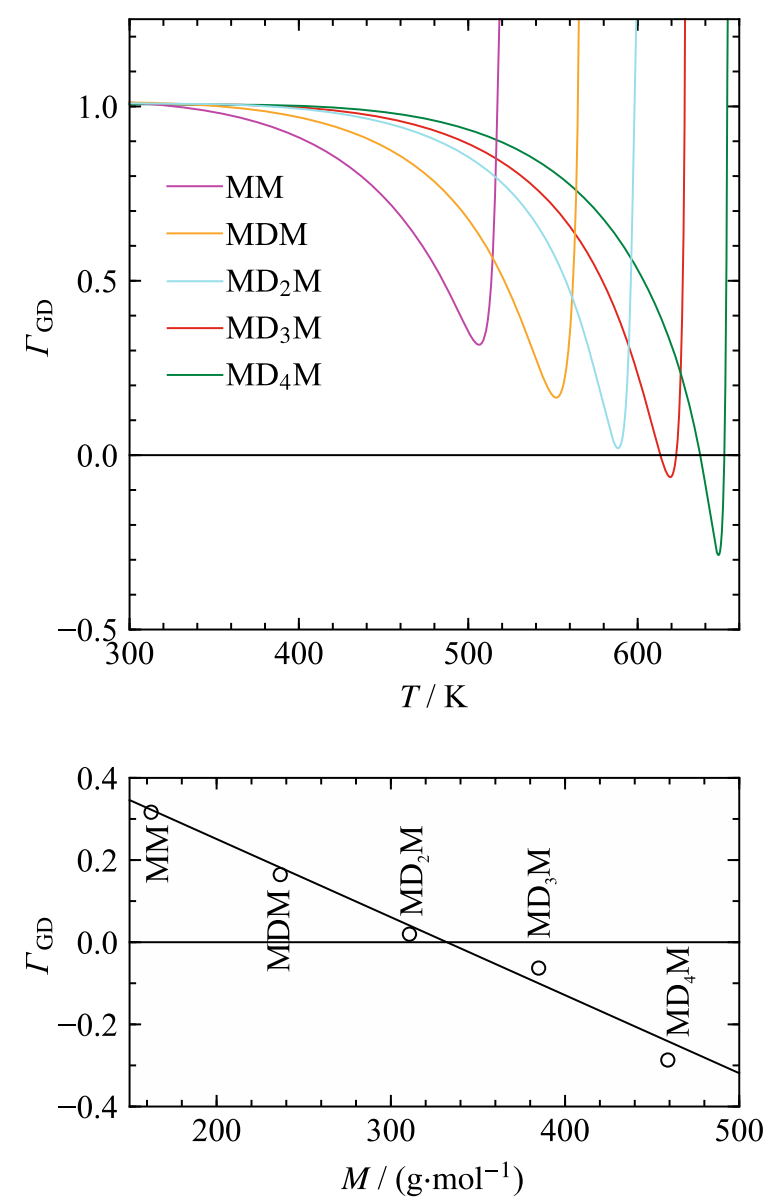

Figure 18. Fundamental derivative of gas dynamics as a function of temperature along the saturated vapor line (top) and as a function of molar mass (bottom) for linear siloxanes. 


\section{CONCLUSION}

New measurements of the liquid density and speed of sound are presented over a wide temperature and pressure range for dodecamethylpentasiloxane $\left(\mathrm{MD}_{3} \mathrm{M}\right)$, tetradecamethylhexasiloxane $\left(\mathrm{MD}_{4} \mathrm{M}\right)$, and decamethylcyclopentasiloxane $\left(\mathrm{D}_{5}\right)$. Based on these data and on other experimental information from the literature, new substance-specific fundamental equations of state in terms of the Helmholtz energy were developed. They can be used to calculate all thermodynamic properties from one correlation for each fluid. The thermodynamic models are valid from the triple point temperature of each fluid up to approximately the critical temperature of each fluid $\left(T_{\max }=630 \mathrm{~K}\right.$ for $\mathrm{MD}_{3} \mathrm{M}$ and $\mathrm{D}_{5}$ as well as $T_{\max }=655 \mathrm{~K}$ for $\left.\mathrm{MD}_{4} \mathrm{M}\right)$. The expected uncertainties of liquid density calculated with the present equations of state are $0.15 \%$, whereas those of the speed of sound in the liquid phase is expected to be $0.2 \%$. The uncertainty of vapor pressure estimations between $380 \mathrm{~K}$ and $550 \mathrm{~K}$ is $0.5 \%$ for all fluids. The accuracy of other properties cannot be assessed due to database limitations. In addition to the best representation of all available experimental data, special attention was paid to the reliable physical and extrapolation behavior of the model. That behavior was continuously monitored during the development of the equations of state and ensured by considering several thermodynamic properties, such as ideal curves, extreme values of thermal properties, heat capacities, speed of sound, etc.

The accuracy of the estimation of caloric properties and of homogeneous liquid density was significantly improved if compared to the equations of state of Colonna et al. ${ }^{14,15}$ through the use of new measurements of these properties. The present models allow, therefore, a more accurate estimation of the values of the fundamental derivative of gas dynamics, which is of paramount importance for the study of non-classical gas dynamic effects. According to these models, $\mathrm{MD}_{3} \mathrm{M}, \mathrm{MD}_{4} \mathrm{M}$, and $\mathrm{D}_{5}$ feature a thermodynamic region in the dense vapor phase where the fundamental derivative of gas dynamics is predicted to be negative.

In the Supplementary Material, fluid files for the use in the software packages TREND ${ }^{73}$ and REFPROP $^{74}$ are provided.

\section{Acknowledgement}

The authors thank M. König and Dr. E. W. Lemmon for their support during the development of the equations of state.

\section{Supporting Information Available}

Text files containing the parameters of the equations are available. For the use in TREND ${ }^{73}$ or REFPROP ${ }^{74}$ they have to be renamed into MD3M.FLD, MD4M.FLD, and D5.FLD. 


\section{REFERENCES}

(1) Owen, M. J. Elastomers: Siloxane. In Encyclopedia of materials: Science and technology; Buschow, K. H. J., Ed.; Elsevier: Amsterdam, 2001; pp 2480-2482.

(2) CES - Silicones Europe. Applications of Siloxanes. https://www.silicones.eu/ (accessed March 20, 2019).

(3) Wacker Chemie AG. Elastomere, Kunststoffe \& Composites. Silicone: Verbindungen und Eigenschaften. https://www.wacker.com/cms/media/publications/downloads/6415_DE.pdf (accessed March 20, 2019).

(4) KEMI Swedish Chemical Agency. Characteristics of Siloxanes. http://webapps.kemi.se/ kemistat/start.aspx?sprak=e. (accessed March 20, 2019).

(5) Zel'dovich, Y. B. Theory of Shock Waves and Introduction to Gas Dynamics; Izdatel'stvo Akademii Nauk SSSR: Moscow, 1946.

(6) Bethe, H. A. On the Theory of Shock Waves for an Arbitrary Equation of State Report No. 545, 1942.

(7) Thompson, P. A. A Fundamental Derivative in Gasdynamics. Phys. Fluids 1971, 14, $1843-1849$.

(8) Colonna, P.; Guardone, A.; Nannan, N. R. Siloxanes: A New Class of Candidate BetheZel'dovich-Thompson Fluids. Phys. Fluids 2007, 19, 086102.

(9) Colonna, P.; Casati, E.; Trapp, C.; Mathijssen, T.; Larjola, J.; Turunen-Saaresti, T.; Uusitalo, A. Organic Rankine Cycle Power Systems: From the Concept to Current Technology, Applications, and an Outlook to the Future. J. Eng. Gas Turbines Power 2015, 137, 100801. (10) Bao, J.; Zhao, L. A Review of Working Fluid and Expander Selections for Organic Rankine Cycle. Renewable and Sustainable Energy Reviews 2013, 24, 325-342.

(11) Preißinger, M.; Brüggemann, D. Thermal Stability of Hexamethyldisiloxane (MM) for High-Temperature Organic Rankine Cycle (ORC). Energies 2016, 9, 183.

(12) Wang, M.; Wang, J.; Zhao, Y.; Zhao, P.; Dai, Y. Thermodynamic analysis and optimization of a solar-driven regenerative organic Rankine cycle (ORC) based on flat-plate solar collectors. Appl. Therm. Eng. 2013, 50, 816-825.

(13) Minea, V. Power generation with ORC machines using low-grade waste heat or renewable energy. Appl. Therm. Eng. 2014, 69, 143-154.

(14) Colonna, P.; Nannan, N. R.; Guardone, A.; Lemmon, E. W. Multiparameter Equations of State for Selected Siloxanes. Fluid Phase Equilib. 2006, 244, 193-211.

(15) Colonna, P.; Nannan, N. R.; Guardone, A. Multiparameter Equations of State for Siloxanes: $\left[\left(\mathrm{CH}_{3}\right)_{3}-\mathrm{Si}-\mathrm{O}_{1 / 2}\right]_{2}-\left[\mathrm{O}-\mathrm{Si}-\left(\mathrm{CH}_{3}\right)_{2}\right]_{i=1, \ldots, 3}$, and $\left[\mathrm{O}-\mathrm{Si}-(\mathrm{CH} 3)_{2}\right]_{6}$. Fluid Phase Equilib. 2008, $263,115-130$.

(16) Span, R.; Wagner, W. Equations of State for Technical Applications. I. Simultaneously Optimized Functional Forms for Nonpolar and Polar Fluids. Int. J. Thermophys. 2003, 24, 139. 
(17) Abbas, R.; Schedemann, A.; Ihmels, C. E.; Enders, S.; Gmehling, J. Measurement of Thermophysical Pure Component Properties for a Few Siloxanes Used as Working Fluids for Organic Rankine Cycles. Ind. Eng. Chem. Res. 2011, 50, 9748-9757.

(18) Thol, M.; Dubberke, F. H.; Rutkai, G.; Windmann, T.; Köster, A.; Span, R.; Vrabec, J. Fundamental Equation of State Correlation for Hexamethyldisiloxane Based on Experimental and Molecular Simulation Data. Fluid Phase Equilib. 2016, 418, 133-151.

(19) Thol, M.; Rutkai, G.; Köster, A.; Dubberke, F. H.; Windmann, T.; Span, R.; Vrabec, J. Thermodynamic Properties for Octamethylcyclotetrasiloxane. J. Chem. Eng. Data 2016, 61, 2580-2595.

(20) Thol, M.; Dubberke, F. H.; Baumhögger, E.; Vrabec, J.; Span, R. Speed of Sound Measurements and Fundamental Equations of State for Octamethyltrisiloxane and Decamethyltetrasiloxane. J. Chem. Eng. Data 2017, 62, 2633-2648.

(21) Dubberke, F. H.; Baumhögger, E.; Vrabec, J. Burst Design and Signal Processing for the Speed of Sound Measurement of Fluids with the Pulse-echo Technique. Rev. Sci. Instrum.

2015, 86, 054903.

(22) Dubberke, F. H.; Rasche, D. B.; Baumhögger, E.; Vrabec, J. Apparatus for the Measurement of the Speed of Sound of Ammonia up to High Temperatures and Pressures. Rev. Sci. Instrum. 2014, 85, 084901.

(23) Anton Paar GmbH. Anton Paar Instruction Manual. DMA HP Density Measuring Cell for High Pressure and High Temperatures; Graz, Austria, 2012.

(24) Wagner, W.; Pruss, A. The IAPWS Formulation 1995 for the Thermodynamic Properties of Ordinary Water Substance for General and Scientific Use. J. Phys. Chem. Ref. Data 2002, 31, 387-535.

(25) Schroeder, J. A.; Penoncello, S. G.; Schroeder, J. S. A Fundamental Equation of State for Ethanol. J. Phys. Chem. Ref. Data 2014, 43, 043102.

(26) Rochow, E. G. Silicium und Silicone: Über steinzeitliche Werkzeuge, antike Töpfereien, moderne Keramik, Computer, Werkstoffe für die Raumfahrt, und wie es dazu kam; Springer Berlin Heidelberg: Berlin, Heidelberg, 1991.

(27) Del Grosso, V. A.; Mader, C. W. Speed of Sound in Pure Water. J. Acoust. Soc. Am. 1972, 52, 1442-1446.

(28) Kroebel, W.; Mahrt, K. H. Recent Results of Absolute Sound Velocity Measurements in Pure Water and Sea Water at Atmospheric Pressure. Acustica 1976, 35, 154-164.

(29) Fujii, K.-i.; Masui, R. Accurate Measurements of the Sound Velocity in Pure Water by Combining a Coherent Phase-Detection Technique and a Variable Path-Length Interferometer. J. Acoust. Soc. Am. 1993, 93, 276-282.

(30) Thol, M.; Dubberke, F. H.; Baumhögger, E.; Span, R.; Vrabec, J. Speed of Sound Measurements and a Fundamental Equation of State for Hydrogen Chloride. J. Chem. Eng. Data 2018, 63, 2533-2547. 
(31) Mohr, P. J.; Newell, D. B.; Taylor, B. N. CODATA Recommended Values of the Fundamental Physical Constants: 2014. J. Phys. Chem. Ref. Data 2016, 45, 043102.

(32) Span, R. Multiparameter Equations of State: An Accurate Source of Thermodynamic Property Data; Springer: Berlin, 2000.

(33) Nannan, N. R.; Colonna, P. Improvement on Multiparameter Equations of State for Dimethylsiloxanes by Adopting More Accurate Ideal-Gas Isobaric Heat Capacities: Supplementary to P. Colonna, N.R. Nannan, A. Guardone, E.W. Lemmon, Fluid Phase Equilib. 244, 193 (2006). Fluid Phase Equilib. 2009, 280, 151-152.

(34) Nannan, N. R.; Colonna, P.; Tracy, C. M.; Rowley, R. L.; Hurly, J. J. Ideal-Gas Heat Capacities of Dimethylsiloxanes from Speed of sound Measurements and ab initio Calculations. Fluid Phase Equilib. 2007, 257, 102-113.

(35) Haar, L.; Gallagher, J. S.; Kell, G. S. The Anatomy of the Thermodynamic Surface of Water: The Formulation and Comparison with Data. Proc. 8th Symp. Thermophys. Prop. 1982, 8, 298-302.

(36) Setzmann, U.; Wagner, W. A New Equation of State and Tables of Thermodynamic Properties for Methane Covering the Range from the Melting Line to $625 \mathrm{~K}$ at Pressures up to 100 MPa. J. Phys. Chem. Ref. Data 1991, 20, 1061-1155.

(37) Span, R.; Wagner, W. A New Equation of State for Carbon Dioxide Covering the Fluid Region from the Triple-Point Temperature to $1100 \mathrm{~K}$ at Pressures up to $800 \mathrm{MPa}$. J. Phys. Chem. Ref. Data 1996, 25, 1509-1596.

(38) Lemmon, E. W.; Jacobsen, R. T. A New Functional Form and New Fitting Techniques for Equations of State with Application to Pentafluoroethane (HFC-125). J. Phys. Chem. Ref. Data 2005, 34, 69-108.

(39) Lemmon, E. W. Numerical Fitting Algorithm for the Development of Equations of State. personal communication, 2018.

(40) Lemmon, E. W.; Overhoff, U.; McLinden, M. O.; Wagner, W. Equation of State for Propylene. to be published in J. Phys. Chem. Ref. Data 2019.

(41) Thol, M.; Rutkai, G.; Köster, A.; Span, R.; Vrabec, J.; Lustig, R. Equation of State for the Lennard-Jones Fluid. J. Phys. Chem. Ref. Data 2016, 45, 023101.

(42) Gao, K.; Wu, J.; Zhang, P.; Lemmon, E. W. A Helmholtz Energy Equation of State for Sulfur Dioxide. J. Chem. Eng. Data 2016, 61, 2859-2872.

(43) Thol, M.; Lemmon, E. W. Equation of State for the Thermodynamic Properties of trans1,3,3,3-Tetrafluoropropene [R1234ze(E)]. Int. J. Thermophys. 2016, 37, 28.

(44) Flaningam, O. L. Vapor Pressures of Poly(dimethylsiloxane) Oligomers. J. Chem. Eng. Data 1986, 31, 266-272.

(45) Rowley, R. L.; Wilding, W. V.; Oscarson, J.; Yang, Y.; Zuendel, N.; Daubert, T.; Danner, R. DIPPR Data Compilation of Pure Chemical Properties; Taylor \& Francis Publishing Company: New York, 2004. 
(46) Alvik, T.; Dale, J.; Fernholt, L.; Hedberg, K.; Schaumburg, K.; Ehrenberg, L. On the Lack of Conformational Preferences for Cyclic Dimethylsiloxane Oligomers. Acta Chem. Scand. 1971, 25, 2131-2141.

(47) Wieser, M. E.; Berglund, M. Atomic Weights of the Elements 2007 (IUPAC Technical Report). Pure Appl. Chem. 2009, 81, 2131-2156.

(48) Fischer, J.; Weiss, A. Transport properties of liquids. V. Self diffusion, viscosity, and mass density of ellipsoidal shaped molecules in the pure liquid phase. Berichte der Bunsengesellschaft für physikalische Chemie 1986, 896-905.

(49) Hurd, C. B. Studies on Siloxanes: I. The Specific Volume and Viscosity in Relation to Temperature and Constitution. J. Am. Chem. Soc. 1946, 68, 364-370.

(50) Lei, Y. D.; Wania, F.; Mathers, D. Temperature-Dependent Vapor Pressure of Selected Cyclic and Linear Polydimethylsiloxane Oligomers. J. Chem. Eng. Data 2010, 55, 58685873.

(51) Mills, A. P.; MacKenzie, C. A. The Application of Bond Parachors to Organosilicon Chemistry. J. Am. Chem. Soc. 1954, 76, 2672-2673.

(52) Palczewska-Tulińska, M.; Oracz, P. Selected Physicochemical Properties of Hexamethylcyclotrisiloxane, Octamethylcyclotetrasiloxane, and Decamethylcyclopentasiloxane. $J$. Chem. Eng. Data 2005, 50, 1711-1719.

(53) Stull, D. R. Vapor Pressure of Pure Substances: Organic Compounds. Ind. Eng. Chem. 1947, 39, 517-540.

(54) Tanaka, T. Heats of Formation of Lower Members of Dimethyl- and Methylisopropoxycyclopolysiloxanes. Bull. Chem. Soc. Jpn. 1960, 33, 282-286.

(55) Thompson, R. 392. Heats of Combustion and Formation of Some Linear Polydimethylsiloxanes; the Si-C and Si-O Bond-Energy Terms. J. Chem. Soc. 1953, 1908-1914. (56) Waterman, H. I.; van Herwijnen, W. E. R.; Den Hartog, H. W. Statistical-Graphical Survey of Series of Linear and Cyclic Dimethylsiloxanes. J. Appl. Chem. 1958, 8, 625-631. (57) Wilcock, D. F. Vapor Pressure-Viscosity Relations in Methylpolysiloxanes. J. Am. Chem. Soc. 1946, 68, 691-696.

(58) Fox, H.; Taylor, P.; Zisman, W. Polyorganosiloxanes...: Surface Active Properties. Ind. Eng. Chem. 1947, 39, 1401-1409.

(59) Golik, A.; Cholpan, P. F. Investigation of Speed of Sound for Some Polysiloxanes. Akusticheskij Zhurnal 1961, 7, 33-39.

(60) Hunter, M. J.; Hyde, J. F.; Warrick, E. L.; Fletcher, H. J. Organo-Silicon Polymers. The Cyclic Dimethyl Siloxanes. J. Am. Chem. Soc. 1946, 68, 667-672.

(61) McLure, I. A.; Barbarin-Castillo, J. M. Orthobaric Liquid Densities for Octamethylcyclotetrasiloxane, Decamethylcyclopentasiloxane, Dimethicone 20, and a Cyclic Poly(dimethylsiloxane). J. Chem. Eng. Data 1994, 39, 12-13. 
(62) McLure, I. A.; Pretty, A. J.; Sadler, P. A. Specific Volumes, Thermal Pressure Coefficients, and Derived Quantities of Five Dimethylsiloxane Oligomers from 25 to $140{ }^{\circ} \mathrm{C}$. $J$. Chem. Eng. Data 1977, 22, 372-376.

(63) Patnode, W.; Wilcock, D. F. Methylpolysiloxanes. J. Am. Chem. Soc. 1946, 68, 358363.

(64) Povey, M. J. W.; Hindle, S. A.; Kennedy, J. D.; Stec, Z.; Taylor, R. G. The Molecular Basis for Sound Velocity in n-Alkanes, 1-Alcohols and Dimethylsiloxanes. Phys. Chem. Chem. Phys. 2003, 5, 73-78.

(65) Schedemann, A. Weiterentwicklung von thermodynamischen Vorhersagemethoden als Grundlage für die Lösungsmittelauswahl bei der Flüssig-Flüssig-Extraktion und der Absorption. Ph.D. thesis, Carl von Ossietzky Universität, Oldenburg, Germany, 2013.

(66) Weissler, A. Ultrasonic Investigation of Molecular Properties of Liquids. III. Linear Polymethylsiloxanes. J. Am. Chem. Soc. 1949, 71, 93-95.

(67) McLure, I. A. Isobaric Heat Capacities of Five Dimethylsiloxane Oligomers at 303.15 and 323.85 K from Sound Velocity and pVT Data. Thermochim Acta 1977, 21, 153-156.

(68) Scott, D. W.; Messerly, J. F.; Todd, S. S.; Guthrie, G. B.; Hossenlopp, I. A.; Moore, R. T.; Osborn, A.; Berg, W. T.; McCullough, J. P. Hexamethyldisiloxane: Chemical Thermodynamic Properties and Internal Rotation about the Siloxane Linkage. J. Phys. Chem. 1961, 65, 1320-1326.

(69) Venkatarathnam, G.; Oellrich, L. R. Identification of the Phase of a Fluid Using Partial Derivatives of Pressure, Volume, and Temperature without Reference to Saturation Properties: Applications in Phase Equilibria Calculations. Fluid Phase Equilib. 2011, 301, 225-233. (70) Arp, V. D.; Persichetti, J. M.; Chen, G.-b. The Grüneisen Parameter in Fluids. J. Fluids Eng. 1984, 106, 193-201.

(71) Herrig, S.; Thol, M.; Harvey, A. H.; Lemmon, E. W. A Reference Equation of State for Heavy Water. J. Phys. Chem. Ref. Data 2018, 47, 043102.

(72) Span, R.; Wagner, W. On the Extrapolation Behavior of Empirical Equations of State.

Int. J. Thermophys. 1997, 18, 1415-1443.

(73) Span, R.; Beckmüller, R.; Eckermann, T.; Herrig, S.; Hielscher, S.; Jäger, A.; Neumann, T.; Pohl, S.; Semrau, B.; Thol, M. TREND. Thermodynamic Reference and Engineering Data 4.0; Lehrstuhl für Thermodynamik, Ruhr-Universität Bochum: Bochum, Germany, 2019.

(74) Lemmon, E. W.; Bell, I. H.; Huber, M. L.; McLinden, M. O. NIST Standard Reference Database 23: Reference Fluid Thermodynamic and Transport Properties-REFPROP, Version 10.0; National Institute of Standards and Technology: Gaithersburg, USA, 2018. 


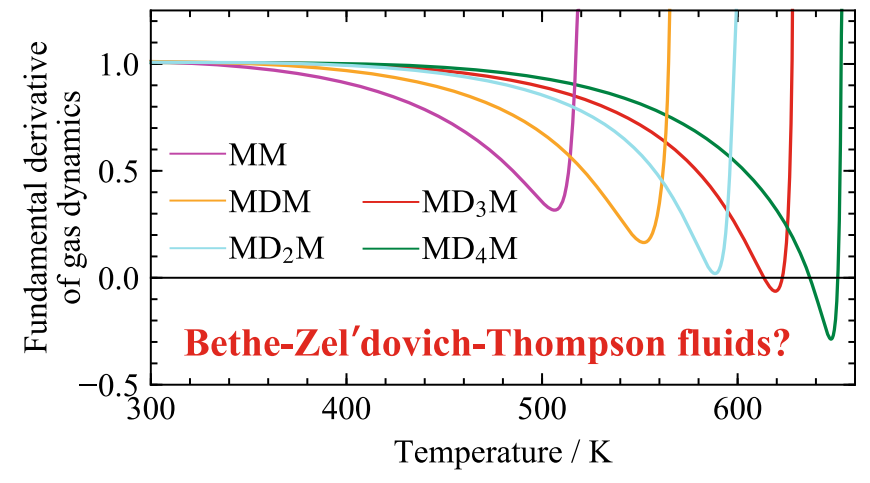


Supporting Information to

\section{Thermodynamic properties of Dodecamethylpentasiloxane, Tetradecame- thylhexasiloxane, and Decamethylcyclopentasiloxane}

Monika Thol $^{*}$, Muhammad Ali Javed ${ }^{2}$, Elmar Baumhögger ${ }^{3}$, Roland Span ${ }^{1}$, Jadran Vrabec ${ }^{2}$

${ }^{1}$ Lehrstuhl für Thermodynamik, Ruhr-Universität Bochum, Universitätsstraße 150, 44801 Bochum, Germany

${ }^{2}$ Thermodynamik und Thermische Verfahrenstechnik, Technische Universität Berlin, Ernst-Reuter-Platz 1, 10587 Berlin, Germany

${ }^{3}$ Lehrstuhl für Thermodynamik und Energietechnik, Universität Paderborn, Warburger Straße 100, 33098 Paderborn, Germany

*E-mail: M.Thol@thermo.ruhr-uni-bochum.de 


\section{DENSITY MEASUREMENTS}

Table 1. Parameters of the calibration equation for the density.

\begin{tabular}{cc|cc|cc}
\hline Parameter & value & Parameter & value & Parameter & value \\
\hline$a$ & 1199.063 & $b_{1} d_{l}$ & -14.842 & $T_{\text {mean }}$ & $39.205^{\circ} \mathrm{C}$ \\
$b_{1}$ & -369.665 & $b_{1} d_{2}$ & -3.121 & width $_{T}$ & 80 \\
$c_{1}$ & -3.169 & $c_{l} d_{2}$ & 1.977 & $p_{\text {mean }}$ & $458.5 \mathrm{bar}$ \\
$d_{1}$ & 782.946 & $d_{l} b_{2}$ & 2.129 & width $_{p}$ & 470 \\
$b_{2}$ & -4.659 & $d_{1} c_{2}$ & 0.308 & $s_{\text {mean }}$ & $2674.868 \mu \mathrm{s}$ \\
$d_{2}$ & 2.905 & $b_{1} c_{l} d_{l}$ & -2.795 & width $_{s}$ & 60 \\
\hline
\end{tabular}

Table 2. Experimental specific density data for $\mathrm{MD}_{3} \mathrm{M}{ }^{a}{ }^{a}$

\begin{tabular}{cccccccc}
\hline$T / \mathrm{K}$ & $p / \mathrm{MPa}$ & $\rho /\left(\mathrm{kg} \mathrm{m}^{-3}\right)$ & $U_{\rho} /\left(\mathrm{kg} \mathrm{m}^{-3}\right)$ & $T / \mathrm{K}$ & $p / \mathrm{MPa}$ & $\rho /\left(\mathrm{kg} \mathrm{m}^{-3}\right)$ & $U_{\rho} /\left(\mathrm{kg} \mathrm{m}^{-3}\right)$ \\
\hline 273.5 & 0.25 & 894 & 1 & 313.0 & 50.15 & 910 & 1 \\
273.5 & 10.05 & 905 & 1 & 313.0 & 60.14 & 918 & 1 \\
273.5 & 20.02 & 915 & 1 & 313.0 & 70.24 & 925 & 1 \\
273.6 & 30.02 & 923 & 1 & 313.0 & 80.49 & 933 & 1 \\
273.5 & 39.95 & 931 & 1 & 313.0 & 89.86 & 939 & 1 \\
273.5 & 50.11 & 939 & 1 & 332.7 & 0.40 & 836 & 1 \\
273.5 & 60.04 & 946 & 1 & 332.7 & 10.11 & 851 & 1 \\
273.5 & 69.99 & 952 & 1 & 332.7 & 20.03 & 864 & 1 \\
273.5 & 80.19 & 959 & 1 & 332.7 & 30.03 & 876 & 1 \\
273.5 & 90.05 & 964 & 1 & 332.8 & 40.15 & 886 & 1 \\
293.2 & 0.36 & 875 & 1 & 332.7 & 50.08 & 896 & 1 \\
293.2 & 10.14 & 887 & 1 & 332.7 & 60.09 & 904 & 1 \\
293.2 & 20.08 & 898 & 1 & 332.7 & 70.08 & 912 & 1 \\
293.2 & 30.12 & 908 & 1 & 332.7 & 80.19 & 920 & 1 \\
293.2 & 40.12 & 916 & 1 & 332.7 & 90.04 & 926 & 1 \\
293.2 & 50.06 & 924 & 1 & 357.4 & 0.39 & 812 & 1 \\
293.2 & 59.96 & 932 & 1 & 357.4 & 9.99 & 829 & 1 \\
293.2 & 70.01 & 939 & 1 & 357.4 & 19.98 & 844 & 1 \\
293.2 & 80.34 & 945 & 1 & 357.4 & 29.96 & 857 & 1 \\
293.2 & 90.51 & 952 & 1 & 357.4 & 40.11 & 868 & 1 \\
313.0 & 0.41 & 856 & 1 & 357.4 & 49.97 & 878 & 1 \\
313.0 & 10.08 & 869 & 1 & 357.4 & 60.07 & 887 & 1 \\
313.0 & 20.09 & 881 & 1 & 357.4 & 70.06 & 896 & 1 \\
313.0 & 30.11 & 892 & 1 & 357.4 & 80.08 & 904 & 1 \\
313.0 & 40.12 & 901 & 1 & 357.4 & 90.03 & 911 & 1 \\
\hline 0.9600 & 1 & 1 & 1 & \\
\hline
\end{tabular}

${ }^{a}$ At a confidence level of $68 \%(k=1)$, the temperature was measured with a standard uncertainty of $u_{T}=0.1 \mathrm{~K}$ and the pressure was measured with an uncertainty of $u_{p}=0.02 \mathrm{MPa}$. The uncertainty of the oscillation period was $u_{\mathrm{s}}=0.03 \mu \mathrm{s}$, and the uncertainty due to calibration was $u_{\text {cal }}=0.5 \mathrm{~kg} \cdot \mathrm{m}^{-3}$. 
Table 3. Experimental specific density data for $\mathrm{MD}_{4} \mathrm{M}{ }^{a}$

\begin{tabular}{|c|c|c|c|c|c|c|c|}
\hline$T / \mathrm{K}$ & $p / \mathrm{MPa}$ & $\rho /\left(\mathrm{kg} \mathrm{m}^{-3}\right)$ & $U_{\rho} /\left(\mathrm{kg} \mathrm{m}^{-3}\right)$ & $T / \mathrm{K}$ & $p / \mathrm{MPa}$ & $\rho /\left(\mathrm{kg} \mathrm{m}^{-3}\right)$ & $U_{\rho} /\left(\mathrm{kg} \mathrm{m}^{-3}\right)$ \\
\hline 273.5 & 0.27 & 911 & 1 & 313.0 & 50.07 & 926 & 1 \\
\hline 273.6 & 10.07 & 921 & 1 & 313.0 & 60.05 & 934 & 1 \\
\hline 273.6 & 20.01 & 931 & 1 & 313.0 & 70.16 & 941 & 1 \\
\hline 273.6 & 30.00 & 939 & 1 & 313.0 & 80.08 & 948 & 1 \\
\hline 273.6 & 40.03 & 947 & 1 & 313.0 & 90.53 & 955 & 1 \\
\hline 273.6 & 50.06 & 955 & 1 & 332.7 & 0.22 & 853 & 1 \\
\hline 273.6 & 60.06 & 961 & 1 & 332.7 & 10.05 & 868 & 1 \\
\hline 273.6 & 70.00 & 968 & 1 & 332.7 & 20.03 & 881 & 1 \\
\hline 273.6 & 80.12 & 974 & 1 & 332.7 & 30.03 & 892 & 1 \\
\hline 273.6 & 89.95 & 979 & 1 & 332.7 & 40.07 & 902 & 1 \\
\hline 293.2 & 0.20 & 891 & 1 & 332.7 & 50.14 & 912 & 1 \\
\hline 293.2 & 10.06 & 903 & 1 & 332.7 & 60.08 & 920 & 1 \\
\hline 293.2 & 20.03 & 914 & 1 & 332.7 & 70.14 & 928 & 1 \\
\hline 293.2 & 30.03 & 923 & 1 & 332.7 & 79.69 & 935 & 1 \\
\hline 293.2 & 39.94 & 932 & 1 & 332.7 & 89.97 & 942 & 1 \\
\hline 293.2 & 50.25 & 940 & 1 & 357.4 & 0.25 & 829 & 1 \\
\hline 293.2 & 60.22 & 947 & 1 & 357.4 & 9.96 & 846 & 1 \\
\hline 293.2 & 70.30 & 954 & 1 & 357.4 & 20.05 & 861 & 1 \\
\hline 293.2 & 80.06 & 961 & 1 & 357.4 & 30.04 & 873 & 1 \\
\hline 293.2 & 90.31 & 967 & 1 & 357.4 & 39.99 & 884 & 1 \\
\hline 313.0 & 0.32 & 872 & 1 & 357.4 & 50.19 & 894 & 1 \\
\hline 313.0 & 10.03 & 886 & 1 & 357.4 & 60.09 & 903 & 1 \\
\hline 313.0 & 20.01 & 897 & 1 & 357.4 & 70.38 & 912 & 1 \\
\hline 313.0 & 30.04 & 908 & 1 & 357.4 & 79.96 & 919 & 1 \\
\hline 313.0 & 40.04 & 917 & 1 & 357.4 & 89.93 & 927 & 1 \\
\hline
\end{tabular}

\footnotetext{
${ }^{a}$ At a confidence level of $68 \%(k=1)$, the temperature was measured with a standard uncertainty of $u_{T}=0.1 \mathrm{~K}$ and the pressure was measured with an uncertainty of $u_{p}=0.02 \mathrm{MPa}$. The uncertainty of the oscillation period was $u_{\mathrm{s}}=0.03 \mu \mathrm{s}$, and the uncertainty due to calibration was $u_{\text {cal }}=0.5 \mathrm{~kg} \cdot \mathrm{m}^{-3}$.
} 
Table 4. Experimental specific density data for $\mathrm{D}_{5}{ }^{a}$

\begin{tabular}{|c|c|c|c|c|c|c|c|}
\hline$T / \mathrm{K}$ & $p / \mathrm{MPa}$ & $\rho /\left(\mathrm{kg} \mathrm{m}^{-3}\right)$ & $U_{\rho} /\left(\mathrm{kg} \mathrm{m}^{-3}\right)$ & $T / \mathrm{K}$ & $p / \mathrm{MPa}$ & $\rho /\left(\mathrm{kg} \mathrm{m}^{-3}\right)$ & $U_{\rho} /\left(\mathrm{kg} \mathrm{m}^{-3}\right)$ \\
\hline 273.5 & 0.39 & 980 & 1 & 313.0 & 50.01 & 994 & 1 \\
\hline 273.5 & 9.98 & 991 & 1 & 313.0 & 59.96 & 1003 & 1 \\
\hline 273.5 & 20.02 & 1001 & 1 & 313.0 & 70.13 & 1011 & 1 \\
\hline 273.5 & 29.96 & 1010 & 1 & 313.0 & 80.65 & 1018 & 1 \\
\hline 273.5 & 40.02 & 1019 & 1 & 313.0 & 90.70 & 1025 & 1 \\
\hline 273.5 & 49.94 & 1026 & 1 & 332.7 & 0.50 & 917 & 1 \\
\hline 273.5 & 59.99 & 1034 & 1 & 332.7 & 9.99 & 933 & 1 \\
\hline 273.5 & 70.07 & 1040 & 1 & 332.7 & 20.12 & 947 & 1 \\
\hline 273.5 & 80.24 & 1047 & 1 & 332.7 & 29.95 & 958 & 1 \\
\hline 273.5 & 90.58 & 1053 & 1 & 332.7 & 40.06 & 969 & 1 \\
\hline 293.2 & 0.42 & 959 & 1 & 332.7 & 49.87 & 979 & 1 \\
\hline 293.2 & 10.08 & 972 & 1 & 332.7 & 60.02 & 988 & 1 \\
\hline 293.2 & 20.32 & 983 & 1 & 332.7 & 70.13 & 996 & 1 \\
\hline 293.2 & 30.31 & 993 & 1 & 332.7 & 80.57 & 1005 & 1 \\
\hline 293.2 & 40.22 & 1002 & 1 & 332.7 & 90.56 & 1012 & 1 \\
\hline 293.2 & 50.20 & 1010 & 1 & 357.4 & 0.53 & 891 & 1 \\
\hline 293.2 & 60.52 & 1018 & 1 & 357.4 & 10.00 & 909 & 1 \\
\hline 293.2 & 70.41 & 1026 & 1 & 357.4 & 20.06 & 925 & 1 \\
\hline 293.2 & 81.34 & 1033 & 1 & 357.4 & 30.07 & 938 & 1 \\
\hline 293.2 & 90.26 & 1039 & 1 & 357.4 & 39.99 & 950 & 1 \\
\hline 313.0 & 0.47 & 938 & 1 & 357.4 & 50.25 & 961 & 1 \\
\hline 313.0 & 10.00 & 952 & 1 & 357.4 & 59.99 & 970 & 1 \\
\hline 313.0 & 20.24 & 965 & 1 & 357.4 & 70.06 & 979 & 1 \\
\hline 313.0 & 30.08 & 976 & 1 & 357.4 & 80.06 & 987 & 1 \\
\hline 313.0 & 40.03 & 985 & 1 & 357.4 & 90.25 & 995 & 1 \\
\hline
\end{tabular}

${ }^{a}$ At a confidence level of $68 \%(k=1)$, the temperature was measured with a standard uncertainty of $u_{T}=0.1 \mathrm{~K}$ and the pressure was measured with an uncertainty of $u_{p}=0.02 \mathrm{MPa}$. The uncertainty of the oscillation period was $u_{\mathrm{s}}=0.03 \mu \mathrm{s}$, and the uncertainty due to calibration was $u_{\text {cal }}=0.5 \mathrm{~kg} \cdot \mathrm{m}^{-3}$.

The combined expanded uncertainty given in Tables 2 to 4 are calculated according to Eq. (4) in the document with the specifications listed in Table 5.

Table 5. Uncertainty budget for the density measurements for the siloxanes at a confidence level of $95 \%(k=2)$

\begin{tabular}{|c|c|c|c|c|}
\hline source & $\begin{array}{c}\text { standard } \\
\text { uncertainty }\end{array}$ & sensitivity coefficient ${ }^{a}$ & $\begin{array}{c}\text { absolute expanded } \\
\text { uncertainty }{ }^{a} /\left(\mathrm{kg} \cdot \mathrm{m}^{-3}\right)\end{array}$ & $\begin{array}{c}\text { relative expanded } \\
\text { uncertainty }{ }^{a} / \%\end{array}$ \\
\hline temperature & $0.1 \mathrm{~K}$ & $\partial \rho / \partial T=0.73 \mathrm{~kg} \cdot \mathrm{m}^{-3} \cdot \mathrm{K}^{-1}$ & 0.15 & 0.016 \\
\hline pressure & $0.02 \mathrm{MPa}$ & $\partial \rho / \partial p=0.09 \mathrm{~kg} \cdot \mathrm{m}^{-3} \cdot \mathrm{MPa}^{-1}$ & 0.02 & 0.002 \\
\hline oscillation period & $0.015 \mu \mathrm{s}$ & $\partial \rho / \partial t=1.29 \cdot 10^{7} \mathrm{~kg} \cdot \mathrm{m}^{-3} \cdot \mathrm{s}^{-1}$ & 0.39 & 0.043 \\
\hline calibration & $0.5 \mathrm{~kg} \cdot \mathrm{m}^{-3}$ & - & 1.00 & 0.111 \\
\hline
\end{tabular}

${ }^{a}$ The values are given for a typical state point at $333 \mathrm{~K}$ and $40 \mathrm{MPa}$.

The absolute expanded uncertainty $u(\rho)=1.08 \mathrm{~kg} \cdot \mathrm{m}^{-3}$ (this value is rounded off to $1 \mathrm{~kg} \cdot \mathrm{m}^{-3}$ in our density data tables) yields a relative expanded uncertainty of $u(\rho)=0.12 \%$. The biggest contribution in the uncertainty is related to the calibration, which also includes the reproducibility and ageing of the measurement cell. 


\section{SPEED OF SOUND MEASUREMENTS}

Table 6. Experimental speed of sound data for $\mathrm{MD}_{3} \mathrm{M}^{a}$

\begin{tabular}{|c|c|c|c|c|c|c|c|}
\hline$T / \mathrm{K}$ & $p / \mathrm{MPa}$ & $w /\left(\mathrm{m} \mathrm{s}^{-1}\right)$ & $U_{w} /\left(\mathrm{m} \mathrm{s}^{-1}\right)$ & $T / \mathrm{K}$ & $p / \mathrm{MPa}$ & $w /\left(\mathrm{m} \mathrm{s}^{-1}\right)$ & $U_{w} /\left(\mathrm{m} \mathrm{s}^{-1}\right)$ \\
\hline 222.24 & 0.123 & 1220.0 & 0.9 & 350.06 & 63.948 & 1170.6 & 0.9 \\
\hline 222.07 & 0.941 & 1224.8 & 0.9 & 350.04 & 80.495 & 1238.1 & 0.9 \\
\hline 222.02 & 2.259 & 1231.7 & 0.9 & 350.03 & 99.003 & 1306.2 & 1.0 \\
\hline 222.00 & 5.352 & 1247.2 & 0.9 & 350.03 & 125.082 & 1392.4 & 1.1 \\
\hline 222.46 & 9.199 & 1264.1 & 1.0 & 399.86 & 0.112 & 646.8 & 0.5 \\
\hline 222.28 & 20.382 & 1315.5 & 1.0 & 399.99 & 0.940 & 656.6 & 0.5 \\
\hline 222.30 & 39.987 & 1395.5 & 1.1 & 399.98 & 2.049 & 669.8 & 0.5 \\
\hline 222.32 & 60.190 & 1468.9 & 1.1 & 399.97 & 4.890 & 701.1 & 0.5 \\
\hline 249.64 & 0.119 & 1113.1 & 0.8 & 399.96 & 9.616 & 747.9 & 0.5 \\
\hline 249.60 & 0.944 & 1118.1 & 0.8 & 399.95 & 20.180 & 835.4 & 0.6 \\
\hline 249.60 & 2.000 & 1124.2 & 0.8 & 399.97 & 41.253 & 971.3 & 0.7 \\
\hline 249.69 & 4.671 & 1139.0 & 0.8 & 399.98 & 60.180 & 1069.0 & 0.8 \\
\hline 249.90 & 9.386 & 1164.0 & 0.9 & 399.98 & 80.019 & 1156.4 & 0.9 \\
\hline 249.89 & 20.126 & 1218.5 & 0.9 & 399.98 & 99.031 & 1230.3 & 0.9 \\
\hline 249.91 & 40.992 & 1310.9 & 1.0 & 400.00 & 125.213 & 1320.6 & 1.1 \\
\hline 249.94 & 59.553 & 1383.1 & 1.1 & 450.21 & 0.154 & 516.8 & 0.4 \\
\hline 249.97 & 71.752 & 1426.6 & 1.1 & 450.20 & 0.951 & 530.0 & 0.4 \\
\hline 250.32 & 76.952 & 1443.4 & 1.1 & 450.19 & 1.980 & 546.0 & 0.4 \\
\hline 300.00 & 0.123 & 938.8 & 0.7 & 450.18 & 4.867 & 586.6 & 0.4 \\
\hline 300.00 & 1.002 & 945.2 & 0.7 & 450.15 & 9.828 & 646.0 & 0.5 \\
\hline 300.02 & 1.976 & 952.3 & 0.7 & 450.15 & 20.030 & 743.3 & 0.6 \\
\hline 300.03 & 5.069 & 974.0 & 0.7 & 450.19 & 40.063 & 886.6 & 0.7 \\
\hline 300.03 & 9.585 & 1004.1 & 0.7 & 450.13 & 62.357 & 1009.1 & 0.7 \\
\hline 300.04 & 20.026 & 1066.9 & 0.8 & 450.14 & 80.600 & 1092.7 & 0.8 \\
\hline 299.99 & 39.397 & 1166.9 & 0.9 & 450.16 & 98.936 & 1166.9 & 0.9 \\
\hline 299.99 & 59.353 & 1254.4 & 0.9 & 450.18 & 124.845 & 1259.5 & 1.0 \\
\hline 300.00 & 79.633 & 1332.5 & 1.0 & 499.83 & 0.118 & 390.1 & 0.3 \\
\hline 300.01 & 99.034 & 1399.7 & 1.1 & 499.84 & 0.976 & 410.1 & 0.3 \\
\hline 300.04 & 124.427 & 1479.2 & 1.2 & 499.83 & 1.987 & 431.5 & 0.3 \\
\hline 350.04 & 0.087 & 785.3 & 0.6 & 499.82 & 4.790 & 482.3 & 0.3 \\
\hline 350.04 & 1.064 & 794.4 & 0.6 & 499.81 & 9.170 & 547.9 & 0.4 \\
\hline 350.04 & 2.102 & 804.0 & 0.6 & 499.74 & 20.208 & 668.7 & 0.5 \\
\hline 350.04 & 4.957 & 829.1 & 0.6 & 499.76 & 40.580 & 825.5 & 0.6 \\
\hline 350.03 & 9.601 & 866.8 & 0.6 & 499.80 & 60.154 & 939.4 & 0.7 \\
\hline 350.03 & 19.944 & 940.3 & 0.7 & 499.78 & 80.284 & 1036.6 & 0.8 \\
\hline 350.04 & 40.114 & 1057.9 & 0.8 & 499.80 & 98.833 & 1114.3 & 0.8 \\
\hline 350.03 & 60.305 & 1154.7 & 0.9 & 499.82 & 123.017 & 1203.6 & 1.0 \\
\hline
\end{tabular}

${ }^{a}$ At a confidence level of $68 \%(k=1)$, the temperature was measured with a standard uncertainty of $u_{T}=0.02 \mathrm{~K}$. Pressure was measured with the following uncertainties $u_{p}: 0.002 \mathrm{MPa}$ for $p<10 \mathrm{MPa}, 0.02 \mathrm{MPa}$ for $p<100 \mathrm{MPa}$ and $0.06 \mathrm{MPa}$ for $p \geq 100 \mathrm{MPa}$. The uncertainty of the time measurement was $u_{\Delta t}=0.002 \mu \mathrm{s}$ and the path length was calibrated with an uncertainty of $u_{\Delta l}=7 \mu \mathrm{m}$. 
Table 7. Experimental speed of sound data for $\mathrm{MD}_{4} \mathrm{M}^{a}$

\begin{tabular}{|c|c|c|c|c|c|c|c|}
\hline$T / \mathrm{K}$ & $p / \mathrm{MPa}$ & $w /\left(\mathrm{m} \mathrm{s}^{-1}\right)$ & $U_{w} /\left(\mathrm{m} \mathrm{s}^{-1}\right)$ & $T / \mathrm{K}$ & $p / \mathrm{MPa}$ & $w /\left(\mathrm{m} \mathrm{s}^{-1}\right)$ & $U_{w} /\left(\mathrm{m} \mathrm{s}^{-1}\right)$ \\
\hline 300.04 & 0.151 & 947.2 & 0.7 & 399.88 & 23.003 & 865.9 & 0.6 \\
\hline 299.99 & 1.227 & 955.1 & 0.7 & 399.89 & 40.438 & 974.2 & 0.7 \\
\hline 299.99 & 2.088 & 961.2 & 0.7 & 399.90 & 60.465 & 1076.0 & 0.8 \\
\hline 299.99 & 5.199 & 982.5 & 0.7 & 399.90 & 80.455 & 1162.5 & 0.9 \\
\hline 299.97 & 9.093 & 1008.0 & 0.7 & 399.91 & 98.823 & 1232.9 & 0.9 \\
\hline 300.06 & 20.092 & 1072.8 & 0.8 & 399.91 & 124.911 & 1321.8 & 1.1 \\
\hline 300.07 & 39.615 & 1171.5 & 0.9 & 450.17 & 0.144 & 539.0 & 0.4 \\
\hline 299.98 & 59.248 & 1256.5 & 0.9 & 450.16 & 0.930 & 551.0 & 0.4 \\
\hline 300.07 & 77.600 & 1326.4 & 1.0 & 450.16 & 1.921 & 565.6 & 0.4 \\
\hline 300.03 & 98.814 & 1399.5 & 1.1 & 450.16 & 4.866 & 604.7 & 0.4 \\
\hline 300.06 & 122.732 & 1473.9 & 1.2 & 450.16 & 9.418 & 657.1 & 0.5 \\
\hline 350.04 & 0.154 & 798.2 & 0.6 & 450.16 & 20.470 & 759.2 & 0.6 \\
\hline 350.04 & 0.897 & 804.9 & 0.6 & 450.18 & 38.909 & 888.5 & 0.7 \\
\hline 350.04 & 1.712 & 812.2 & 0.6 & 450.18 & 60.139 & 1004.8 & 0.7 \\
\hline 350.05 & 4.907 & 839.5 & 0.6 & 450.17 & 80.370 & 1097.1 & 0.8 \\
\hline 350.05 & 9.500 & 875.8 & 0.6 & 450.18 & 99.043 & 1171.6 & 0.9 \\
\hline 350.05 & 20.069 & 949.1 & 0.7 & 450.20 & 125.078 & 1263.4 & 1.0 \\
\hline 350.04 & 40.457 & 1065.3 & 0.8 & 499.72 & 0.118 & 420.8 & 0.3 \\
\hline 350.01 & 46.672 & 1096.2 & 0.8 & 499.70 & 0.954 & 437.9 & 0.3 \\
\hline 350.02 & 60.822 & 1160.9 & 0.9 & 499.66 & 1.744 & 453.4 & 0.3 \\
\hline 350.04 & 79.990 & 1238.9 & 0.9 & 499.63 & 4.824 & 506.8 & 0.4 \\
\hline 350.04 & 98.619 & 1306.9 & 1.0 & 499.57 & 9.313 & 569.3 & 0.4 \\
\hline 350.04 & 122.855 & 1386.4 & 1.1 & 499.71 & 19.050 & 671.8 & 0.5 \\
\hline 399.96 & 0.116 & 663.4 & 0.5 & 499.71 & 39.904 & 831.1 & 0.6 \\
\hline 399.95 & 0.688 & 670.1 & 0.5 & 499.71 & 60.461 & 948.9 & 0.7 \\
\hline 399.87 & 1.804 & 683.1 & 0.5 & 499.71 & 81.589 & 1048.6 & 0.8 \\
\hline 399.85 & 4.940 & 716.4 & 0.5 & 499.68 & 98.136 & 1116.9 & 0.8 \\
\hline 399.80 & 9.112 & 756.4 & 0.5 & 499.70 & 125.138 & 1215.1 & 1.0 \\
\hline 399.94 & 19.584 & 841.3 & 0.6 & & & & \\
\hline
\end{tabular}

${ }^{a}$ At a confidence level of $68 \%(k=1)$, the temperature was measured with a standard uncertainty of $u_{T}=0.02 \mathrm{~K}$. Pressure was measured with the following uncertainties $u_{p}: 0.002 \mathrm{MPa}$ for $p<10 \mathrm{MPa}, 0.02 \mathrm{MPa}$ for $p<100 \mathrm{MPa}$ and $0.06 \mathrm{MPa}$ for $p \geq 100 \mathrm{MPa}$. The uncertainty of the time measurement was $u_{\Delta t}=0.002 \mu \mathrm{s}$ and the path length was calibrated with an uncertainty of $u_{\Delta l}=7 \mu \mathrm{m}$. 
Table 8. Experimental speed of sound data for $\mathrm{D}_{5}{ }^{a}$

\begin{tabular}{|c|c|c|c|c|c|c|c|}
\hline$T / \mathrm{K}$ & $p / \mathrm{MPa}$ & $w /\left(\mathrm{m} \mathrm{s}^{-1}\right)$ & $U_{w} /\left(\mathrm{m} \mathrm{s}^{-1}\right)$ & $T / \mathrm{K}$ & $p / \mathrm{MPa}$ & $w /\left(\mathrm{m} \mathrm{s}^{-1}\right)$ & $U_{w} /\left(\mathrm{m} \mathrm{s}^{-1}\right)$ \\
\hline 249.29 & 0.239 & 1100.9 & 0.8 & 399.94 & 0.974 & 639.9 & 0.5 \\
\hline 249.57 & 1.037 & 1104.3 & 0.8 & 399.94 & 2.025 & 652.0 & 0.5 \\
\hline 249.36 & 1.993 & 1110.5 & 0.8 & 399.94 & 4.829 & 681.8 & 0.5 \\
\hline 249.30 & 5.101 & 1127.5 & 0.8 & 399.94 & 9.783 & 728.9 & 0.5 \\
\hline 249.29 & 9.631 & 1151.3 & 0.9 & 399.93 & 19.973 & 810.5 & 0.6 \\
\hline 249.30 & 19.880 & 1201.0 & 0.9 & 399.92 & 40.038 & 937.4 & 0.7 \\
\hline 249.27 & 40.209 & 1288.8 & 1.0 & 399.93 & 60.030 & 1038.3 & 0.8 \\
\hline 249.27 & 59.540 & 1362.0 & 1.0 & 399.93 & 80.225 & 1125.0 & 0.8 \\
\hline 249.27 & 79.415 & 1430.3 & 1.1 & 399.94 & 99.565 & 1198.4 & 0.9 \\
\hline 299.91 & 0.114 & 919.4 & 0.7 & 399.94 & 124.537 & 1282.7 & 1.0 \\
\hline 299.90 & 1.039 & 926.0 & 0.7 & 450.02 & 0.142 & 499.5 & 0.4 \\
\hline 299.90 & 1.981 & 932.5 & 0.7 & 450.02 & 0.966 & 512.7 & 0.4 \\
\hline 299.90 & 4.992 & 952.8 & 0.7 & 450.02 & 1.953 & 527.5 & 0.4 \\
\hline 299.90 & 9.866 & 984.1 & 0.7 & 450.02 & 4.911 & 568.0 & 0.4 \\
\hline 299.88 & 19.873 & 1042.9 & 0.8 & 450.01 & 9.677 & 623.4 & 0.4 \\
\hline 299.87 & 39.676 & 1140.8 & 0.9 & 450.02 & 20.378 & 722.5 & 0.6 \\
\hline 299.88 & 61.012 & 1231.0 & 0.9 & 450.00 & 40.230 & 860.2 & 0.6 \\
\hline 299.89 & 80.266 & 1302.9 & 1.0 & 450.01 & 60.538 & 969.6 & 0.7 \\
\hline 299.89 & 98.929 & 1367.1 & 1.0 & 450.01 & 80.008 & 1057.6 & 0.8 \\
\hline 299.90 & 124.791 & 1446.8 & 1.2 & 450.01 & 98.651 & 1131.5 & 0.8 \\
\hline 350.10 & 0.117 & 768.5 & 1.1 & 450.01 & 124.659 & 1222.5 & 1.0 \\
\hline 350.10 & 0.997 & 776.9 & 1.0 & 499.98 & 0.176 & 372.6 & 0.3 \\
\hline 350.10 & 2.087 & 786.1 & 0.9 & 499.98 & 0.972 & 391.1 & 0.3 \\
\hline 350.10 & 4.872 & 809.5 & 0.8 & 499.98 & 2.065 & 414.2 & 0.3 \\
\hline 350.10 & 9.632 & 846.7 & 0.8 & 499.97 & 4.920 & 464.8 & 0.3 \\
\hline 350.10 & 20.073 & 918.5 & 0.7 & 499.97 & 9.842 & 534.3 & 0.4 \\
\hline 350.10 & 39.960 & 1030.1 & 0.6 & 499.96 & 20.016 & 641.9 & 0.5 \\
\hline 350.10 & 60.176 & 1124.6 & 0.6 & 499.91 & 40.608 & 797.2 & 0.6 \\
\hline 350.11 & 79.704 & 1203.6 & 0.6 & 499.95 & 60.054 & 907.9 & 0.7 \\
\hline 350.13 & 98.211 & 1270.1 & 0.6 & 499.95 & 79.934 & 1001.7 & 0.7 \\
\hline 350.12 & 124.614 & 1355.6 & 0.6 & 499.94 & 98.621 & 1078.4 & 0.8 \\
\hline 399.94 & 0.121 & 629.9 & 0.5 & 499.96 & 124.388 & 1171.2 & 1.0 \\
\hline
\end{tabular}

${ }^{a}$ At a confidence level of $68 \%(k=1)$, the temperature was measured with a standard uncertainty of $u_{T}=0.02 \mathrm{~K}$. Pressure was measured with the following uncertainties $u_{p}: 0.002 \mathrm{MPa}$ for $p<10 \mathrm{MPa}, 0.02 \mathrm{MPa}$ for $p<100 \mathrm{MPa}$ and $0.06 \mathrm{MPa}$ for $p \geq 100 \mathrm{MPa}$. The uncertainty of the time measurement was $u_{\Delta t}=0.002 \mu \mathrm{s}$ and the path length was calibrated with an uncertainty of $u_{\Delta l}=7 \mu \mathrm{m}$. 
The combined expanded uncertainty given in Tables 6 to 8 are calculated according to Eq. (6) in the document with the specifications listed in Table 9.

Table 9. Uncertainty budget for the speed of sound measurements for the siloxanes at a confidence level of $95 \%$ $(k=2)$.

\begin{tabular}{|c|c|c|c|c|c|}
\hline source & type & $\begin{array}{l}\text { measuring } \\
\text { range }\end{array}$ & $\begin{array}{c}\text { standard } \\
\text { uncertainty }\end{array}$ & sensitivity coefficient ${ }^{a}$ & $\begin{array}{c}\text { relative expanded } \\
\text { uncertainty }{ }^{a} \%\end{array}$ \\
\hline \multirow[t]{2}{*}{$\begin{array}{l}\text { tempera- } \\
\text { ture }\end{array}$} & PT-25 & $84-693 \mathrm{~K}$ & $0.02 \mathrm{~K}$ & $\partial w / \partial T=2.2 \mathrm{~m} \cdot \mathrm{s}^{-1} \cdot \mathrm{K}^{-1}$ & 0.008 \\
\hline & Keller PAA-33X & $<10 \mathrm{MPa}$ & $0.002 \mathrm{MPa}$ & & \\
\hline \multirow[t]{2}{*}{ pressure } & Keller PAA-33X & $<100 \mathrm{MPa}$ & $0.01-0.02 \mathrm{MPa}$ & $\partial w / \partial p=0.58 \mathrm{~m} \cdot \mathrm{s}^{-1} \mathrm{MPa}^{-1}$ & 0.011 \\
\hline & Honeywell TJE & $<200 \mathrm{MPa}$ & $0.06 \mathrm{MPa}$ & & \\
\hline time & handyscope HS5 & - & $0.002 \mu \mathrm{s}$ & $\partial w / \partial t=5.6 \cdot 10^{7} \mathrm{~m} \cdot \mathrm{s}^{-2}$ & 0.021 \\
\hline $\begin{array}{l}\text { path } \\
\text { length }\end{array}$ & - & - & $7 \mu \mathrm{m}$ & $\partial w / \partial \Delta l=5.3 \cdot 10^{4} \mathrm{~s}^{-1}$ & 0.070 \\
\hline
\end{tabular}

${ }^{a}$ The values are given for a typical state point at $350 \mathrm{~K}$ and $40 \mathrm{MPa}$. 